\title{
28. GALAXIES
}

PRESIDENT: V. C. Rubin

VICE-PRESIDENT: P. C. van der Kruit

ORGANIZING COMMITTEE: S. d'Odorico, J. Einasto, J.A. Graham, P.W. Hodge,

I.D. Karachentsev, D. Lynden-Bell, A. Toomre, M.-H. Ulrich, K.-I. Wakamatsu,

B.E. Westerlund

The present Report covers the period 1982-1984. As with previous Reports, it has not been possible to cover all publications or even every field of research. Some subsections of this Report, the Magellanic Clouds for example, report a body of work as extensive as that in some commissions.

This Report is divided into eleven sections, and has been prepared by the President, Vice-President, members of the organizing committee, and chairpersons of the working groups. References are given by commonly used abbreviations for journals, or by reference numbers in Astronomy and Astrophysics Abstracts, in which summarles of the papers are found. The symbol + is used in place of et al.

1. GALAXIES IN GENERAL (B.E. Westerlund)

\section{Survey work; Catalogues}

The "ESO/Uppsala Survey of the ESO(B) Atlas" is completed and published (Lauberts, ESO). It contains 18,438 non-stellar objects, of which 16,000 are galaxies south of $-17 \% 5$. Magnitudes and radial velocities are given for 2102 galaxies. $B$ and $R$ magnitudes are being measured for all galaxies. A "Merged Catalogue of Galaxies" has been compiled on magnetic tape at the Abastumani Astrophysical Observatory, and is to be permanently updated (Bull Inf CDS, Strasbourg, No. 25). It contains data on 31,917 galaxies north of $-33^{\circ}$ and brighter than $15 . \mathrm{m}_{1}$. The data have been collected from the MCG, the UGC, the RCBG, and the CGCG catalogues and include positions, magnitudes, dimensions, morphological types, radial velocities and radio continuum fluxes.

Duboist report the extension of the data base SIMBAD to galaxies. Three catalogues are included: the Uppsala General Catalogue of Galaxies (UGC), the Morphological Catalogue of Galaxies (MCG) and the ESO/Uppsala Survey of the ESO(B) Atlas. Positions (1950), magnitudes and dimensions for 45,317 galaxies are included. The next steps will be to add the Catalogue of Markarian Galaxies, the Second Reference Catalogue of Bright Galaxies, and a list of quasars.

Longo+ (Univ Tex Monogr Astron No. 3) have presented a general catalogue of 3578 galaxies brighter than $\mathrm{V}=16^{\mathrm{m}}$. It contains information on photoelectric magnitudes and UBV colors published during 1962-1982. E. Sadier has presented new optical and radio data for 248 southern $E$ and So galaxles (AJ $89,23,34,53$ ). Radial velocities, photoelectric magnitudes (169 new), and the result of a radio continuum survey at 2.7 and $5.0 \mathrm{GHz}$ (38 detections) are included. A list of the "Second Byurakan Spectral Survey" has been given by Markarian and Stepanyan (Astrof 1z 19, 639). 109 galaxies and QSO candidates brighter than $20^{\mathrm{m}}$ were found in a region $4^{\circ}$ by $4^{\circ}$ centered on $\alpha=11^{h} 30^{m}, \delta=+59^{\circ}$. A bibliography of the surface photometry of galaxies has been published by Davoust and Pence (AA Suppl 49, 631). For 650 galaxies from more than 300 references, it contains methods of observation and reduction, photometric information and limiting surface brightness. A catalogue of 2251 quasars with published redshifts and of 554 Seyfert and related galaxies known prior to Sept. 1983, has been published by Veron-Cetty and Veron (ESO Sci Rep 
No. 1). It includes positions, redshifts, photometry and 6 and $11 \mathrm{~cm}$ flux densities.

Images in the Gunn $r$-band have been obtained with the Palomar $1.52 \mathrm{~m}$ telescope of fields around 108 bright quasars (ApJ Suppl 54, 495; ApJ 280, 79). The catalogue gives positional offsets, magnitudes and classifications into stars or quasars. An analysis of the quasar and control fields suggests that quasars are associated with galaxies at their cosmological redshifts. Brosche reports on a "List of Elite Galaxies" (Tartu Teated No. 68). The aim is to provide a first approximation of a generally agreed data base and to make the "black holes" in that data obvious.

Rudnicki reports that at the Jagelionian University Observatory the general catalogues of galaxies are surveyed. Atmospheric extinction, visibility of galaxies on plates, and the effect of interstellar extinction in terms of galactic and supergalactic coordinates are examined. A search for intergalactic obscuring clouds in selected areas is in progress. Intergalactic clouds may be located in volumes of space with a scarce population of galaxies. Magnitudes, colours, ellipticities and position angles have been complled for 6724 galaxies in a $2: 63$ area centered on the Coma cluster (MN 202, 113). Kraan-Korteweg has presented a catalogue of a complete sample of 180 Virgo cluster galaxies (AA Suppl 47, 505), within $6^{\circ}$ of M87 and brighter than $B_{T}=14.0$. Positions, morphological types, magnitudes and radial velocities are given. Lucey+ (MN 203, 545) present redshifts, morphological classifications, orientations and ellipticities for 129 galaxies within a $6^{\circ} \times 6^{\circ}$ region in Horologium-Reticulum. The prominent clusters in the region form a supercluster with a mean redshift of $18,000 \mathrm{~km} \mathrm{~s}^{-1}$. Cranet (The Birth of the Universe, p. 325) are surveying galaxies in Horologium for radial velocities and photoelectric photometry.

Pesch and Sanduleak are producing a low-dispersion objective prism survey of the northern galactic polar region. Two parts have been published (ApJ Suppl 51 , 1711 ; 55, 517). 196 galaxies and 179 unresolved objects (possible QSO candidates) are reported, all being blue and/or emission line objects with $11^{\mathrm{m}}<\mathrm{B}<18^{\mathrm{m}}$. Kinman reports on an objective-prism survey for emission-line galaxies with the CTIO Curtis Schmidt and the KPNO Burrell Schmidt (MN 202, 53). Particular care is taken to ensure completeness and to get confirming spectroscopic IIDS scans (8A resolution) and BV photoelectric photometry. This confirming work is about $75 \%$ complete. Moss and Whittle are using the Burrell Schmidt to conduct an Ha-survey for emission-line galaxies in nearby Abell clusters ( $\mathrm{cz}<9500 \mathrm{~km} \mathrm{~s}^{-1}$ ). Data for four clusters have so far been obtained. 10-15\% of the galaxies listed in the Zwicky CGCG catalogue are detected in Ha-emission.

An attempt to find the optimal two-dimensional classification system for spiral galaxies has been described by Whitmore (ApJ 278, 61) on the basis of a large number of observational parameters. Studies of polar-ring galaxies have been presented by Wakamatsu+ (PASP 95, 2983; ApJ 273, 167; AJ 87, 980).

Courtes reports that a balloon survey in UV (2000A) has been carried out for the nearest galaxies as a joint program of the Laboratoire d'Astronomie Spatiale of Marseille and the Geneva Observatory. Excellent photographs of M33, M31 and M81 reveal the hot stellar populations and show close correlations with the faint diffuse $\mathrm{Ha}$ general emission, as well as the dust emission observed by IRAS. A new UV survey will start in Sep. 1984 with a new $40 \mathrm{~cm}$ telescope (FOCA-1000).

A near-IR photographic atlas of 54 spiral galaxies has been presented by D.M. Elmegreen (ApJ Suppl 47, 229). The sample was subdivided into categories of grand design and flocculent spirals. A 12-division morphological system has been developed by D.M. and B.G. Elmegreen (MN 201, 1021) and applied to the classification of 305 nearby galaxies. The system has been applied to 829 spiral galaxies in 22 clusters ( $M N 201,1035$ ) and to 261 low-inclination galaxies in 
groups (ApJ 267, 31). "A Catalogue of Infrared Magnitudes and HI Velocity Widths for Nearby Galaxies" has been published by Aaronsont (ApJ Suppl 50, 241). It supplies integrated $\mathrm{H}(1.6 \mu)$ magnitudes, HI velocity widths and velocities, positions, types, inclinations, diameters, HI flux density integrals and distances.

Some results from the IRAS survey have been published. The IRAS observations of 165 galaxies in the Revised Shapley-Ames Catalogue led to the detection of 108 predominantly spiral galaxies (ApJ 278, L67). In the IRAS mini-survey 86 galaxies were detected at $60 \mu$ (ApJ 278, L71), again many spirals.

A new "Catalogue of Galaxy Redshifts" (CGR) has been compiled containing known redshifts for -4000 galaxies (ApJ Suppl 49, 111). Davis reports that a redshift survey has been completed at the Harvard-Smithsonian Center for Astrophysics. The catalogue contains radial velocities for a magnitude-limited sample of 2400 galaxies within a solid angle of 2.7 steradians (IAU Symp 104, 167). The sample is complete to $\mathrm{Z}$ wicky magnitude $=14.5$.

A catalogue of rotation curves for 271 galaxies is given by Baiesi-Pillastrine+ (AA Suppl. 53, 373) with bibliographical information from 332 references from before 1982 .

\section{Measurements in the Radio and X-ray Regions}

Heeschen reports observations of 226 extragalactic radio sources made at 9 and $6 \mathrm{~cm}$ at Green Bank (IAU Symp 97) and of 34 sources at $20 \mathrm{~cm}$ and $11 \mathrm{~cm}$ made at Arecibo. This is a part of an investigation of the global properties of variability of an almost complete sample of extragalactic radio sources. Compact sources exhibit variability on scales of 2 to 20 days with a mean amplitude of $2 \%$. Whether this is intrinsic or the result of interstellar or circumsource scintillation is unclear. Observations to investigate variations on time scales of 30 seconds to 2 days and 20 days to 6 years are under way.

Sherwood reports that 464 Markarian galaxies with UV-excess from 1 ists 7-11 have been observed at $11 \mathrm{~cm}$ with the Effelsberg $100 \mathrm{~m}$ telescope. 13 detections were made with flux densities $\geq 30 \mathrm{mJy}$, a number appreciably less than expected from earlier observations of gälaxies from lists 1-5.

Total power and polarization maps of NGC 253 have been obtained at $10.7 \mathrm{GHz}$ (AA 127,177 ) according to a report from Urbanik. He also reports mappings of NGC 520, NGC 2276, NGC 3448, NGC 3267 and four bright Virgo cluster galaxies (NGC 4254, 4231, 4501 and 4569). Among these only the interacting galaxy NGC 2276 showed an extremely high disk emission, indicating very intense star formation.

A resurvey of the $\pm 4^{\circ}$ declination zone of the Parkes $2700 \mathrm{MHz}$ survey has been made with the Parkes $64 \mathrm{~m}$ telescope. The catalogue of 822 sources is now complete to $0.25 \mathrm{Jy}$ and all but one source have been measured at $5 \mathrm{GHz}$. 59 new optical identiflcations have been made (Austr J Phys 35, 177, 207). Results for 294 bright galaxies observed with the Molonglo Radio Telescope at $408 \mathrm{MHz}$ are given in Austr $\mathrm{J}$ Phys 35, 321. Radio luminosity functions are derived as well as spectral indices for 73 galaxies observed at other frequencies.

The Very Large Array (VLA) has been extensively used. 602 radio sources from the $5 \mathrm{GHz}$ MIT-Green Bank Survey have been observed (ApJ 278, L95) and their radio

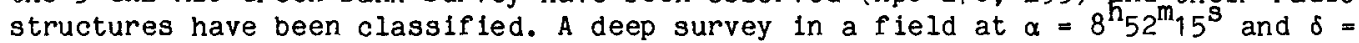
$+17^{\circ} 16^{\prime}$ has been carried out at $1.4 \mathrm{GHz} .159$ sources stronger than 84 HJy were found and 45 have been identified with optical objects brighter than $B=21.5$. Another deep survey is described in Science 225, 23. Many VLA observations study QSO's, BL Lac objects and nuclei of galaxies (MN 208, 231; ApJ 279,93; 280, 476; AJ $89,5,189,195,203,753,932 ;$ AA 134, 207). 
VLBI observations with the NASA Deep Space Network (Goldstone-Madrid and Goldstone-Tidbinbilia) at $2.29 \mathrm{GHz}$ surveyed 257 extragalactic radio sources within $10^{\circ}$ of the ecliptic (AJ 89,336 ), to find compact radio sources which form an accurate reference frame for planetary spacecraft navigation. Components with sizes smaller than 2.5-3.2 milliarcsec and with flux densities $>0.1 \mathrm{Jy}$ were found in 144 sources.

The Bologna Northern Cross radio telescope investigated at $408 \mathrm{MHz}$ the low-frequency spectral index distribution of a complete sample of 308 extragalactic sources north of $\delta=-10^{\circ}$ (AJ 89, 323). From a sample of 132 bright elliptical galaxies a strong correlation between radio activity and flattening is found (MN $206,899)$. No ellipticals with axial ratios below 0.65 are found to be radio emitters.

The Westerbork Radio Synthesis Telescope has observed a large sample of so galaxies (AA 106, 183) at $1415 \mathrm{MHz}$. Continuum properties were compared with those of $\mathrm{S}$ and $\mathrm{E}$ galaxies. Many investigations at the HI $21 \mathrm{~cm} I$ ine wavelength have been published. "A General Catalogue of $21 \mathrm{~cm}$ Line Data" (AA Suppl 47, 171) includes 1207 galaxies. Corrected $21 \mathrm{~cm}$ line widths at three different levels are given together with HI fluxes and radial velocities. "A General Catalogue of HI Observations of External Galaxies" (ESO Sci Prepr No. 250, 176) has been compiled from pre-1983 data. It contains information for 4000 galaxies and is more than $95 \%$ complete for references and more than $98 \%$ complete for entries up to the deadline. A $21 \mathrm{~cm}$ line survey of 359 galaxies of types so to $\mathrm{Im}$, south of $\delta=-18^{\circ}$, has been carried out with the Parkes $64 \mathrm{~m}$ telescope. 196 galaxles were detected and their spectra and integrated properties have been presented (AA Suppl 50, 451).

Mirabel reports on HI surveys of 91 Seyfert galaxies (ApJ 277, 92) and 80 spirals with strong nuclear radio sources (ApJ 260,$75 ; 270, \mathrm{~L} 35$ ) undertaken with the Arecibo $305 \mathrm{~m}$ telescope. In Seyfert galaxies, redshifts from nuclear optical emission lines are systematically smaller than systemic velocities derived from HI Iine profiles. A $21 \mathrm{~cm}$ line survey of a magnitude-limited sample of interacting and isolated galaxies has been carried out at NRAO and Arecibo (ApJ Suppl 53, 269). 166 interacting and 113 isolated galaxies were observed. The radio data were combined with optical data on blue diameters, axial ratios and magnitudes. A HI survey has been made of galaxies in UMa and CVn using the Mk IA $76 \mathrm{~m}$ telescope at Jodrell Bank (MN 201, 1073). New accurate systemic velocities, HI masses and total masses are given. The region contains possibly four associations of galaxies.

Benn+ (MN 200, 747; 209,683) have conducted a deep optical/radio survey near the North Galactic Pole, the 5C 12 survey: at 408 and $1407 \mathrm{MHz}$ with the Cambridge One-Mi le telescope, at $4.85 \mathrm{GHz}$ with the $100 \mathrm{~m}$ Effelsberg telescope, and in the optical with the 48" Schmidt telescope of Hale Observatorles. 299 radio sources were detected at $408 \mathrm{MHz}$ within a $4^{\circ}$ diameter circle and 65 at $1407 \mathrm{MHz}$ within a $1^{\circ}$ diameter circle. The optical part (MN 206, 475) presents 1200 objects brighter than $\mathrm{m}_{\mathrm{v}}=22.5$ in 1 flelds centered on the radio positions.

HI and optical parameters are presented for 151 southern low surface brightness galaxies (LSB) selected from UK Schmidt plates and observed with the Parkes $64 \mathrm{~m}$ telescope (MN 200, 325). The $21 \mathrm{~cm}$ line was detected in 100 of the galaxies. The data are consistent with the LSB being of systematically lower mass than brighter galaxies of same type and linear dimensions. Observations have been made at Arecibo of 325 isolated galaxies to define a standard sample for the comparison of the HI content of galaxies in different intergalactic environments (AJ 89,758 ). HI emission was detected in 288 cases with a definite increase of the non-detection rate for the earliest types.

A catalogue of HI diameters of 238 galaxies has been compiled from 98 new measurements at Nancay, and 140 previously published results from Nancay and 
Arecibo (AA Suppl 55, 55). The catalogue has been used to study HI expansions and relations between gaseous and stellar extensions in galaxies (AA 122, 273). HI fluxes, optical and infrared luminosities and broad-band colors of 300 spiral galaxies in clusters are presented by Bothun+ (preprint) together with a discussion of a number of derived parameters. The Tully-Fisher relation has been the subject of a number of studies (Bottinellit, AA 118; 4; ApJ 254,8;280,34; and Aaronsont ApJ 265, 1; 279, 475).

A review of molecular clouds in external galaxies appears in Ann Rev Astron Astrophys 20, 517. The proceedings of a workshop on "Extragalact ic Molecules" (Publications Div., NRAO) held at Green Bank is published. D.M. and B.G. Elmegreen report on ${ }^{12} \mathrm{CO} 2.6 \mathrm{~mm}$ observations of $23 \mathrm{spiral}$ galaxies with the NRAO $36 \mathrm{ft}$ radio telescope (AJ 87, 626). 4 SAB galaxies, NGC 157, 2903, 4321 and 5248, and the Seyfert galaxy NGC 1068 were detected. For further investigations in the radio region the reader is referred to the Report of Commission 40.

An X-ray survey with the Einstein Observatory of a complete sample of $403 \mathrm{CR}$ radio galaxies has been made by Fabblano+ (ApJ 277, 115). 26 galaxjes were detected in $x$-rays, having $x$-ray luminosities between $10^{41}$ and $10^{45} \mathrm{ergs} \mathrm{s}^{-1}$. The authors found a correlation between $X-r$ ay Iuminosity, $5 \mathrm{GHz}$ nuclear source luminosity, strong optical emission line spectra and the presence of double radio morphology. They suggest a nuclear or $1 g$ in of the $X-r a y$ emission.

Long has discussed $X$-ray properties of normal galaxies as derived from observations with the Einste in Observatory of more than 50 nearby normal galaxies (Adv Space Res Vol 2, 177). X-ray luminosities were $-2 \times 10^{-4}$ of optical luminosities and showed no strong correlation with morphological type. Nuclei of some normal galaxies were found to be luminous $X-r a y$ sources. Many $X-r$ ay observations have been devoted to galaxies with active nuclei and QSO's. See the relevant subsection in this Report and also the Reports of Commissions 44 and 48 .

\section{STRUCTURE AND EVOLUTION OF GALAXIES}

(P.C. van der Kruit)

One recent IAU meeting was devoted to the structure and evolution of galaxies, IAU Symp 100: "Internal Kinematics and Dynamics of Galaxies" (33.012.004). However, relevant discussions took place in other meetings: "Extra-galactic Molecules" (31.012.048), "Morphology, and Dynamies of Galaxies" (Saas-Fee; 32.012.032), "Origin and Evolution of Galaxies" (33.012.023), "Kinematics, Dynamics and Structure of the Milky Way" (33.012.016), "Evolution in old Stellar Populations" (in 33.012.028), "The Nearby Stars and the Stellar Luminosity Function" (34.012.065) and "The Milky Way Galaxy" (IAU Symp 106; to be published). In this Report, nuclei and active galaxies are not included. These subjects are covered at other places in this commission Report and in the Reports of commissions 40 and 48 .

\section{Elliptical Galaxies}

DYNAMICS. Models of early type galaxies have been reviewed in detail by Binney (Ann.Rev.20, 399; 32.151.048; 33.151.052). Emphas is is placed on calculation of individual stellar orbits in bullding models and in studying their propertles; this follows the work by Schwarzschlld (see his older chapter 30.158.103). Models are usually tri-axial, such as that of Wilkinson and James (31.151.012) or de Zeeuw and Merritt (33.151.093). A numerical simulation of Schwarzschild's model shows it to be "rugged and robust" $(31.151 .058)$. Petrou has introduced a third integral to construct oblate models with reasonable rotation curves $(33.151 .014 / 15)$. P1smis and Moreno (preprint) have found that an initially oblate stellar system oscillates between oblate and prolate form. 
Lake and Norman (34.151.001) considered both stellar orbits and gas motions and showed how stable orbits of the gas may exist in planes tilted with respect to the symmetry axis of the triaxial potentials. Such motions may explain "spindle" systems like NGC 2685. In ordinary $E$ and $S 0$ galaxles the gas motions appear different and the velocity dispersions lower than in the stars (AJ 89, 356).

Tonry (33.151.012) has used models of elliptical galaxies with constant M/L ratio but with anisotropic velocity distributions to show that their projected properties on the sky fit actual observations. Binney and Mamon (31.158.320) show that the observed kinematics of $M 87$ can be fit with a model of constant M/L. Although the models suggest a tight star cluster in the inner regions, there is no need to suppose the presence of a black hole of $5 \times 10^{9} \mathrm{M}$. Efstathiou+ (32.158.267) model their observations of NGC 5813 with an increasing velocity anisotropy with radius combined with a very modest increase in $\mathrm{M} / \mathrm{L}$.

One interpretation of the $X-r a y$ observations of $M 87$ points to a dark halo with a mass of a few times $10^{13} \mathrm{M}$ within $200 \mathrm{kpc}$ (33.158.109). The opposite conclusion of no dark halo and $5 \times 10^{11^{\circ} \mathrm{M}}$ within $100 \mathrm{kpc}$ has however been reported previously $(30.158 .007)$ and in that case matter beyond $200 \mathrm{kpc}$ does exist but is assoclated with the Virgo cluster. The dark mass is associated with M87 rather than with the cluster.

Daviest (33.157.029) have measured rotation and central veloctty dispersion in faint ellipticals. These rotate more rapidly compared to the velocity dispersion than bright ellipticals do and are almost consistent with the predictions provided by oblate models supported by rotation (see also 33.157.097). This agrees with expectations based on formation by merging or fragmentation in pancakes. Lake (33.151.006) finds that tidal torques make the same prediction.

For analytical purposes Jaffe $(33.157 .041)$ has proposed a simple formula to describe the three-dimensional light distribution in spherical galaxies. Van Albada (32.151.065) has given stellar dynamical collapse models that produce luminosity profiles like observed galaxies; abundance gradients are probably conserved through violent relaxation and so do not force us to conclude that the collapse was dissipational. Colour gradlents have been studied by Wirth and Shaw (30.158.063; $33.157 .037)$ and Borosont $(34.157 .123)$, who find rounder isochromes than isophotes at small radii, probably supporting dissipationless collapse or merger as the formation process.

GLOBULAR CLUSTERS. Frogel (ApJ 278, 119) has measured infrared colours of clusters in NGC 5128 and found that these are old; some have higher metallicities than the sun. Comparison with Virgo ellipticals shows that the mean metallicity may be a function of the parent galaxy's luminosity. Hesser+ (ApJ 276, 491) also observed clusters in NGC 5128 . One conclusion is that the velocities indicate a mass of $1.6 \times 10^{12} M_{\odot}$ within $36 \mathrm{kpc}$.

SHELLS. Discovery of shells in the light distribution in the outer parts of elliptical galaxies has led to a major southern hemisphere survey, a catalog of 137 galaxies by Malin and Carter (34.157.147). Photometry of one shell in NGC 1344 shows that it is made of stars and is consistent wi th a (young) disk population (31.158.058). Schweizer (33.157.105), who calls these features ripples, argues that they arise from a merger of an elliptical and a disk galaxy. Quinn (ApJ 279, 596) has modeled such encounters with numerical simulations and derives information on the form of the potential. This suggests a massive dark halo.

GAS AND STELLAR CONTENT. Mapping of HI in large elliptical galaxies is now revealing its kinematics and distribution (ApJ 246,708; Knapp and Raimond, preprint). The gas appears to have originated externally via accretion or merging rather than resulting from mass loss in stellar evolution or ejection from the 
nucleus (Knapp, 33.157.100). UV observations have revealed the presence of a component of hot stars (32.158.228). These are most easily, but not unambiguously, interpreted as a small population of recently formed stars (32.158.192/194; Veron and Veron-Cetty, preprint).

CCD's make it possible to study the stellar content of dwarf ellipticals in the Local Group. Observations of NGC 947 (34.157.027) and NGC 205 (ApJ 278, 575) are reported. Besides improved metallicities and distances, these studies give information on possible intermedlate-age components from a study of the asymptotic giant branch. No such component is detected in excess of $10 \%$ of the stars observed. The majority of stars have ages similar to globular clusters. This contrasts with a similar study in BV of the Corina dwarf spheroidal (34.157.108) for which the bulk of stars indlcate an intermediate age of about $7 \mathrm{Gyr}$. There may actually be no population II at all.

Clusters of galaxies at large redshift have more blue galaxies than present day clusters. This Butcher-Oemler effect has recently been confirmed by its discoverers in a cluster at $z=0.38$ (Nature 310,31 ). Recent deep counts in two colours by Shanks+ (MN 206, 767) provides evidence that this effect also occurs outside of clusters.

\section{Disk Galaxies}

PHOTOMETRY OF BULGES. Wirth and Shaw $(30.158 .063 ; 33.157 .037)$ measured $(B-V)$ colour gradients in bulges as a function of luminosity and morphology. Pure spheroids have small gradients but show less correlation with luminosity of the bulge. Griersmith+ $(32.158 .018)$ have found gradients In the near infrared in ( $\mathrm{V}-\mathrm{K})$ and $(J-K)$ as well as in (U-V) but these are smaller than those in $E$ and So galaxies. Colors are similar in spiral bulges and ellipticals, suggesting similar populations. The strong bulge Sab system NGC 7814 has a measurable abundance gradient; its HI rotation curve (31.158.309) indicates an M/L gradient.

KINEMATICS OF BULGES. Dramatic developments have occurred in this field. The main conclusions by Kormendy and Illingworth (31.158.1551; 33.157.024), Whitmore and K1rshner (30.151.051) and Schechter (34.157.041) are that in contrast to giant ellipticals but similar to ellipticals of comparable luminosities, bulges appear flattened mainly by their rotation. Like ellipticals they exhibit a relation in which the bulge luminosity is proportional to a power between 4 and 8 of the velocity dispersion. Box-shaped bulges are cylindrical rotators and bulges in barred galaxies appear to be rapidly rotating triaxial structures (31.158.193). Jarvis (preprint) has constructed dynamical models in which bulges are flattened by rotation; these models represent the photometry and observed kinematics in bulges. The So-galaxy NGC 3115 has a spiral-like bulge flattened by rotation (31.158.156). Dressler and Sandage $(33.157 .026)$ find the same result in a sample of 32 mostly so-galaxies. These follow the luminosity-velocity dispersion relation. So's have central velocity dispersions intermediate between those of ellipticals and spirals; this argues against recent stripping of present-day spirals to form so-galaxies. The diffuse component of the spindle galaxy NGC $4650 \mathrm{~A}$ has a higher rotation velocity than central velocity dispersion (ApJ 277, 526). Reviews of much of these matters have been presented at IAU $100(33.151 .051 / 52)$. A detailed dynamical model of the bulge of M31 has been presented by McElroy (34.157.028) and Lawrie $(34.157 .111)$ finds the bulge velocity dispersion from planetary nebulae to be similar to that of the stellar population.

GLOBULAR CLUSTERS. Searches for globular clusters have been made in a number of sp irals: NGC 891 and 4565 (31.154.015); M33 (32.158.032); NGC 55 (32.154.015; 33.157.002), NGC $253(33.157 .014)$ ), NGC 4594 (ApJ 89, 216) and NGC 2403 (AA 130, 162). The analysis of NGC 4594 by three Harrisses indicates that spirals have fewer globular clusters per unit spherold light than ellipticals (see also 30.151 .064 ). 
Cohent (ApJ 281, 141) find that the clusters in M33 have an age range similar to the LMC and unlike larger spirals.

ROTATION CURVES AND MASSES. Observations, systematics and analyses of optical and radio rotation curves have been presented and reviewed by Rubin ( 32.158 .125 ; 33.157.058; 33.157.246; ApJ 289) and Bosma (30.158.239/40; 33.157.059); see also Lequeux (AA 125, 394). There must be dark matter of at least the mass of the luminous material and maybe constituting $90 \%$ of all mass in the universe. Interpretation of the rotation curves needs to take the disk and spheroid contributions into account (Bahcall+, 31.151.078).

The shape of the rotation curve out to about the Holmberg radius can be used to estimate the halo distribution. Carignan and Freeman (preprint) use pure-disk systems and show that their rotation curves can be modelled excellently by a disk with constant $M / L$ plus an isothermal sphere. The latter contains at least half the mass within the last measured point. Analyses of rotation curves of NGC 5907 by Casertano (33.157.118) and NGC 891 by Bahcall (33.157.119) give comparable results. A different approach is to use both stellar and gaseous $k$ inematics in the z-direction in the disks to estimate the disk density distributions. Van der Kruit and co-workers (33.157.071; ApJ 278, 81; AA 132, 20) show that out to the optical edge the disks contain no more than $1 / 3$ to $1 / 2$ of the total mass.

Total masses out to larger radii are derived by sampling the gravitational field by the following methods: (i) The HI rotation curve of NGC 3198 has now been extended to more than 2 Holmberg radil (Bergemant preprint) and still falls to decilne; (ii) HI absorption at a similar distance from NGC 3067 against the QSO 3C232 would suggest a still rising rotation curve according to Rubint (31.158.141); (iii) Polar rings may be used to estimate the potential (33.157.246; AJ 89, 618, 975); (iv) The velocities of companions or in binary galaxles can be examined (e.g. van Moorsel, 34.157.038/39). All studies indicate large amounts of non-luminous matter; only the study of the barred system NGC 3992 (32.158.094) comes to a different conclusion.

The nature of the dark matter is not clear. Several studies (30.158.176; $31.158 .330 ; 34.157 .114$ ) conclude that faint $M$ stars would provide too much luminosity or are locally too scarce to provide sufficient mass. The search for dark matter in dwarf galaxies (22.157.032/033), and the velocity dispersions of globular clusters in Fornax (34.157.030) or carbon stars in Draco (33.157.031) do not yet allow a definitive conclusion. Lake and Schommer (ApJ 279, L19) claim large masses in binary dwarf galaxies.

Burstein (31.158.032) has critically discussed the Tully-Fisher relation between luminosity and rotation speed, while Tully+ (31.158.212) have discussed a colour-magnitude relation. Burstein and Sarazin (33.157.008) propose a simple model that explains the distribution of global properties among spirals. A model for the formation of disk galaxies including a dark halo has been given by Jones and Wyse $(33.151 .065)$.

STRUCTURE OF THE DISK. Boroson (30.158.059) determined radial luminosity profiles for 26 spirals and finds that the bulge-to-disk ratio is related to Hubble type with a scatter larger than observational uncertainty. His sample displays a constant extrapolated central surface brightness of the disk but with a larger scatter than Freeman's original sample. Meisels and Ostriker (preprint) argue that the Hubble type is independent of disk but is only related to the absolute magnitude of the bulge.

The vertical structure of the stellar disk was further studied by van der Kruit and Searle $(31.158 .038)$ in terms of their three-dimensional model with constant thickness with radius. The observational result was confirmed in NGC 4565 
by Jensen and Thuan (32.158.307). A very modest increase of thickness with radius $(-10 \%)$ is consistent with the data $(32.151 .008)$. Hamabe and Okamura (31.158.248; 32.158.211) also decompose edge-on galaxies into bulge and disk. Van der Kruit and Freeman (ApJ 278, 81) measure high stellar z-dispersions in the central parts of two disks in agreement with the predictions of constant thickness. Mechanisms to explain the age-velocity dispersion relation in the solar neighbourhood have been investigated $(31.151 .018 ; 33.151 .070 ; 33.151 .103 ; 34.115 .006 / 089)$, but no definitive conclusion regarding the actual process has appeared.

The thickness of the HI layer can provide information on the mass distribution in the disk. The HI velocity dispersion of $7-10 \mathrm{~km} \mathrm{~s}^{-1}$ measured in face-on systems by van der Kruit and Shostak (31.158.079, AA 132, 20, AA 134, 258) leads to an M/L of the old disk population $(33.157 .071$, AA 134, 258). Van der Kruit and Freeman (preprint) have observed that the vertical velocity dispersion in the old disk of NGC 5247 declines in accordance with the constant thickness. M/L in the old disk is $6 \pm 2$ in $B$.

ABUNDANCE GRADIENTS AND CHEMICAL EVOLUTION. Radial gradients in chemical abundances have been deduced from observations in many systems (31.158.037; $30.158 .135 ; 31.158 .065)$. McCall's extensive observations for 20 galaxies (33.157.256) have significantly added to the available data. Chemical evolution has been reviewed by Pagel (30.151.022). Noteworthy is also the deduction by Rayo+ (31.158.065) that the pregalactic He abundance was $Y_{p}=0.216 \pm 0.01(3 \sigma)$.

SPIRAL STRUCTURE AND STAR FORMATION. TOomre $(30.151 .021)$ has described the swing amplifier as the basis of spiral structure in galaxies. Some aspects of density wave theory have been studied by Bertin and Haas (31.151.040, see also 34.151.038). Self-propagating star formation as the bas is of spiral structure has been revlewed by Seiden and Gerola (31.151.084). Observationally, Elmegreen and Elmegreen (32.158.268/9; ApJ Suppl 54,127) have concentrated on "flocculent" spiral galaxies. Studies in the near IR show in the latter an absence of structure in the older disk populations, and these galaxies appear not to display a density wave. Observationally the situation is the opposite in grand-design spirals (e.g. M51), while M33 and M101 are examples of intermediate cases which display evidence for both phenomena. Freedman and Madore (ApJ 280, 592) stress the non-uniqueness of self-propagating spirals in their model simulations. A comprehensive review of all aspects involving spiral structure has been written by Athanassoula (Physics Report, in press).

Kennicutt has studied the relation of spiral arm structure and morphology with classification schemes and model predictions $(30.158 .241 ; 31.158 .026 / 71)$. A significant addition to models for the star formation rate in galaxies has been provided also by Kennicutt (34.157.068) who includes integrated $\mathrm{Ha}$ emission together with UBV photometry as input. These constraints better disentangle the effects of star formation rate and initial mass function. Spirals in the Virgo cluster indicate a lower star formation rate $(33.157 .111)$. A similar approach by de Giola-Eastwood+ (ApJ 278, 564) estimates the radial dependence of the present star formation rate in NGC 6946; it turns out strongly correlated with the $\mathrm{H}_{2}$-surface density. Correlations between physical properties in HI-HII associations have been discussed in detall by Viallenfond+ (32.158.249). Hodge and Kennicutt (33.157.163) have studied the radial distribution of HII regions as a function of Hubble type and luminosity of the galaxy. Wevers (Thesis, Groningen) found that colour indices in spiral disks are approximately constant with radius. This implies that the time-dependence of the star formation rate has not been a strong function of galactocentric distance.

MOLECULES. The last three years have seen an enormous increase in the study of CO in external systems (see 33.157.064/65). This compound traces the distribution of $\mathrm{H}_{2}$. Many major spirals have been studied $(31.158 .340 / 45 ; 32.158 .011$; 
33.157.013/051/239, ApJ 276, 476). The Co follows the distribution of blue light with radius as first pointed out by Young and Scoville (32.15.011).

BARRED GALAXIES. The gas exhibits strong streamings in most systems $(31.158 .011 / 14 ; 32.158 .007 ; 34.157 .048 ;$ MN 207, 9). The narrow dust lanes in bars are coincident with ridges of radio continuum emission (33.157.242) and thus are almost certainly strong shocks. The general morphology and stellar kinematics has been reviewed and observed by Kormendy (30.158.104; 331.57.089; 34.157.187). Many studies examine orbits of stars and gas flows in barred potent1als (31.151.033; $32.151 .033 / 35 / 80 ; 34.151 .040)$. Strong deviations from circular rotation are predicted and have been confirmed in general. Tight spiral features in nuclei are described as the loci of matter ejected from the rotating nucleus (Pismis and Moreno, Astrofizica 20, 7; preprint).

STABILITY. Sellwood $(33.151 .039)$ has reviewed various criteria for disk stability; prominent among these is that of Toomre for local stability. Global stability can be provided by a dark halo if it contains sufficlent mass or if the central disk has a large velocity dispersion (also 33.151.040). The halo masses reported above are sufficient to provide stability for the disks according to the criterion of Efstahiout $(31.151 .081)$. Van der Kruit and Freeman (preprint) find that the central velocity dispersion in NGC 7184 is also high, about twice that required as minimum by Toomre's local criterion out to about 2 scalelengths. This agrees with numerical simulations by Sellwood and Carlberg (ApJ 282). Kormendy (preprints) has observed the stellar kinematics in the disks of an SO and SBO system. These are very hot and there seems a clear correlation between high velocity dispersions and absence of structure in disks.

RADIO EMISSION. Radio continuum emission from galaxy disks contains a thermal (HII regions) and non-thermal component. Observations show in many cases a steeper radio spectrum with increasing radius. This cannot be entirely explained by a varying thermal/non-thermal ratio $(31.158 .016 / 069 / 111 ; 34.157 .074 / 125 ; A A 135,213)$ as was thought earlier. Polarization to map magnetic fields is now feasible (31.158.085; AA 135,213$)$. So galaxies usually have much fainter radio disks than spirals $(31.158 .086)$. Individual sources can often be related to HII-regions or supernova remnants (e.g. 31.158.018; 33.157.006). The general distribution of non-thermal emission does not show concentrations towards regions of recent star formation $(34.157 .074 ; 31.158 .016 / 111)$. However, the integrated $H \alpha-f l u x$ correlates well with the non-thermal flux density and Kennicutt interprets this to mean that the old disk population is not important in the generation of relativistic electrons and cosmic rays $(33.157 .114)$.

Edge-on spirals show much thicker disks in radio continuum than the HI or young population (AA 133,1 ) so cosmic rays may diffuse in the $z$-direction. In NGC 891 Allen and $\mathrm{Hu}$ (preprint) find a close correspondence with the optical surface brightness. Parker's instability may play an important role in establishing the z-structure of the radio disks (see also 34.157 .105 ).

WARPS. The theories of warps have most recently been reviewed by Toomre $(33.151 .036)$. Several models involving hydrodynamic phenomena related to interactions between a rotating disk and non-rotating halo (30.151.059; $31.151 .025)$, corrugations (30.151.0031), triaxial potentials (33.151.090) or self-gravity (MN 206, 691) have been published. A particularly simple suggestion is that warps have to do with the shape and tilt of a massive dark halo (33.151.037, ApJ 280,117 ).

IRAS. Infrared observations with IRAS have been published in ApJ 278 Letters (March 1, 1984). M31 has emission associated with the dust, HI, HII and radio continuum in a ring of radius about $10 \mathrm{kpc}$. Most Shapley-Ames galaxies are detected. However in an (infrared) flux limited sample the infrared to blue 
luminosity ratio varies by a factor 100 .

ENVIRONMENTAL INFLUENCES. Schweizer has reviewed the observational evidence for mergers among galaxies (31.158.007; 33.157.105). Villumsen (31.151.041; $34.151 .008)$ and White $(33.151 .059$, also 34.151 .057$)$ have presented numerical simulations of merging galaxies. The final remnants show some characteristics similar to actual galaxies. The effects of cluster environment on the gas content of galaxies has been studied by Sullivan and co-workers (30.158.060/61; $31.158 .323 / 24)$. Most clusters do not contain preferentially gas-deficient spirais. One example of a major spiral outside large clusters that appears presently to undergo stripping is NGC 1961 (32.158.248). HI mapping in so-galaxies has shown that current stripping is not a major process among these galaxies (33.157.077).

\section{GROUPS AND CLUSTERS OF GALAXIES (J.A. Graham)}

This field has been very active during the past three years; stimuli have come from a varlety of sources. New photographic sky surveys are available, such as the ESO/SRC Atlas which provides a first deep view over the whole area of the southern sky. New detectors, such as the CCD, allow us to carry out area photometry of galaxies to a greater precision than possible previously (Schneider+, ApJ 264, 337; $268,476)$, and multiple galaxy integrated photometry within a single frame is being explored (Bautz+, ApJ 255, 57). Optical fibre devices (Hintzen+, AJ 87, 1656) enable spectra to be obtained of many objects within a small field thus raising the efficiency of data collection during valuable large telescope time. Larger and faster computers have added to our theoretical understanding of gravitational interactions within groups and clusters of galaxies. Perhaps the principal difficulty confronting workers in the field is the need to find time to digest and interpret the vast amount of new data which is coming in.

\section{The Local Group}

Galaxies within the Local Group are easily resolved into individual stars and are baslc to the calibration of the distance scale. Cepheid variables have been studled in M33 (Sandage and Carlson, ApJ 267, L5) and in NGC 6822 and IC 1613 (McAlary+ ApJ 273,539; 276, 487). CCD detectors are especially useful for accurate stellar photometry (Walker, MN 209, 83). Hoessel and collaborators (ApJ 254, 68; 271,$65 ; 274,577$ ) and Christian and Tully (AJ 88, 934) have applied the technique with success to several dwarf galaxies of the Local Croup. These data will be essential for interpreting the integrated spectra of more distant galaxies. Low dispersion spectroscopic studies have led to the identification of carbon stars in the Local Group (Richer and Westerlund, ApJ 264, 114) while Moffet and Shara (ApJ 273, 544) as well as Massey and Conti (ApJ 273, 576; BAAS 16, 12) have detected and studied individual wolf-Rayet stars. The mass and dynamics of the Local Group have been examined by Einasto and Lynden-Bell (MN 199, 67). The motion of the Local Group with respect to a backdrop of distant galaxies has been determined by Hart and Davies (Nature 297, 191).

\section{Nearby Groups}

A useful all-sky catalog of nearby galaxy groups has been published by Huchra and Geller (ApJ 257, 423), based on a parameter-space search for density enhancements. A remarkable one-to-one correspondence is found between this computerized search and the earlier catalog of de Vaucouleurs. A second catalog, by Geller and Huchra (ApJ Suppl 52,61), uses a simflar search procedure on the deeper, northern hemisphere CFA redshift survey. A search of the Palomar Sky Survey by Hickson (ApJ 255, 382) has led to the identification of 100 compact groups. Optical velocities have been obtained by Huchra, integrated 21 -cm profiles by $B$. 
Williams and Rood, and rotation curves by Rubin, Ford, and Schechter.

The galaxy group in Sculptor, which is the nearest to our own, is important to the distance scale calibration. Its member galaxies extend over a distance of 1 Mpc or more along the line-of-sight (Graham, ApJ 252, 474). Webster and Smith (MN 204, 743) have observed HII regions to determine abundance gradients over the faces of the Sculptor galaxies NGC 55,253, 300 and 7793 as well as for the Centaurus galaxies M83 and NGC 5253. Carignan (ApJ Supp1, in press) presents three color surface photometry for NGC 247, 300 and 7793 as part of a larger study of the propertles of disk galaxies. Photometric sequences have been published by Alcaino and Liller (AJ 89, 814).

A survey for new dwarf galaxies in the M81/M82 group has been carried out by Borgnen and Karachentseva (AN 303, 189). Borgnen+ also give photometrlc data for 39 dwarf galaxies in the area (AN 303, 287). Recent work by Sandage (AJ 89, 621; 89, 630) shows that the galaxies which have been regarded as members of the group may also spread over a large radial distance range. More than one group of galaxies may be involved.

Elmegreen and Elmegreen (MN 201, 1021; ApJ 267, 31) study the relative frequency of grand design spiral structure features among galaxles in groups and in the general field. They find a significant trend towards higher fractions of spirals with grand design features when the galaxy has companions or has a bar feature. However it seems that the presence or lack of bars or companions do not in themselves determine uniquely the occurrence of grand design features.

Correlations between luminosities, velocity dispersions and virlal radii for 49 Huchra-Geller groups have been analysed by Menzett+ (AA 125, 368). A similar sample has been examined by Mardirossiant (AA 126, 86). They find no evidence for merging. Danks and Materne (AA, in press) suggest that the Klemola 25 group forms a ring structure which resembles model simulations of a materlal $r$ ing undergoing bead instabilities. The mass of the system is equivalent to that of a giant elliptical galaxy. Schneider+ (ApJ 273, L1) have discovered an intergalactic HI cloud in the western part of the Leo group of galaxies. Its nominal mass is about $10^{9} \mathrm{M}$. The NGC 1961 group of galaxies has been studied by Cottesman+ (MN 202, 21P). They conclude that a large amount of unseen matter is associated either with NGC 1961 itself or with the galaxy group as a whole.

Sulentic reports that a sample of 196 companion galaxies in 60 spiral-dominated groups show a significant positive redshift excess. A second sample of 62 companions in $21 \mathrm{E} / \mathrm{SO}$ dominated groups show no excesses. A third study with Arp was of a redshift sample obtained in a $21 \mathrm{~cm}$ survey (AJ 88, 489). An analysis by Sulentic and Lorre of VV172 and Seyfert's Sextet (AA 120, 36) reveals evidence for physical and unusual structural and color properties. $21 \mathrm{~cm}$ mapping of Stephan's Quintet (AJ 88, 2671) suggests that the discordant redsh1ft galaxy in that group interacts with its neighbors. A statistical analysis of the frequency of interacting quartets contradicts the findings of Rose (ApJ 211,311 ) and suggests that the above three groups are not chance optical configurations (ApJ 270, 417).

\section{Clusters of Galaxies}

Clusters of galaxies are the largest known bound aggregates of matter in the Universe and much of observational cosmology depends on our understanding of their basic properties. The subject was well covered at IAU Symposium 104, Early Evolution of the Universe and its Present Structure. Superclustering is covered in the Report of Commission 47.

Abel1's cluster catalog (ApJ Suppl 3, 211) continues as a fundamental reference. At the time of his premature death, Abell was working with Corwin on a 
southern extension using the ESO/SRC Sky Atlas. The Abe11-Corwin survey is based on the deep J survey photographs. A search for distant, highly red-shifted clusters is being carrled out by West and collaborators (Rev Mex Astron Astrophys 6, 35; IAJ, 15, 25). Deep CCD frames are being obtained for photometric calibration and for the identification of faint cluster members. A useful table of redshifts for 339 Abell clusters is given by Sarazint (AA 108, L7). Two color photometry for $17 \mathrm{rich}$ clusters of galaxies has been published by Butchert (ApJ Suppl 52, 183). The CFA redshift survey by Huchrat (ApJ Suppl 52, 89) contains the best avallable radial velocities for 2401 galaxies brighter than $M_{B}=14.5$. Geller reports that several studies are in progress to examine the role of substructure in rich clusters (PASP 94, 421; ApJ 257, 23). Substructure can provide clues to the cluster's dynamical age and can affect the derived mass/Iight ratios. A complete velocity survey revealed that the Cancer cluster is not real but consists of an unbound collection of groups.

The nearest clusters of galaxies reveal the most about content, structure and evolution. An ESO Workshop, "The Virgo Cluster of Galaxies", was taking place as this Report was being prepared. In Virgo, we can study the dwarf galaxies in detail and estimate their contribution to the mass of the cluster. Reeves (ApJ SuppI 53, 375) has published a new catalog of Virgo dwarf galaxies. He feels (BAAS 15, 946) that an appreciable mass contribution from the dwarfs cannot be ruled out. Reeves is also investigating whether dust, swept out of the now dustless $E$ and So galaxles, can be detected from the reddening of background objects. A major series of papers on the Virgo cluster has been initiated by Sandage and collaborators (AJ $89,64 ; 89,919)$. Particular attention has been given so far to the morphology of the dwarf galaxies. Caldwell (AJ 88, 804) and Caldwell and Bothun (ApJ 280, 528) have been studying structure and stellar content of the Virgo dwarfs. A complete sample of 180 Virgo cluster galaxies has been isolated by Kraan-Kortweg (AA Suppl 47, 505). These lie within $6^{\circ}$ of $M 87$ and are brighter than $B=14.0$. Positions, morphologlcal types, magnitudes and velocities are provided. Radial velocities for 100 Virgo galaxies are given by Karachentsev and Karachentseva (Pis'ma Astron Zh 8 , 198) who Include many faint blue objects which appear to be members of the Virgo cluster. Dressler (ApJ 281, 512) has used new, high quality spectra for 23 galaxies in Virgo and 30 in Coma to derive luminosity-velocity dispersion and luminosity-metallicity relations. These are then used to estimate the difference in distance modulus between the VIrgo and Coma clusters.

The extent and deficiency of HI in spiral galaxies in Virgo has been studied by Glovanel11 and Haynes (AJ 88, 881) and by Giovanadit (ApJ 267, 35). Stauffer (ApJ 264,14) finds no difference between emission line strengths for the Virgo galaxies and a fleld sample.

The dynamics of nearby galaxy clusters are being analysed with the improved data base. Tully and Shaya (ApJ 281, 31) have studied the infall of galaxies belonging to the Virgo southern extension into the main body of the cluster. They find that the influx of gas-rich systems is sufficiently large that all spirals and ellipticals presently observed in Virgo appear to have arrived on a time scale of only $1 / 3$ to $1 / 2$ of the age of the Universe. Rich clusters of galaxies which have attained a high degree of symmetry, such as Coma, are ideal objects for studying structure and long-term evolutionary trends. Codwint (MN 207, 113) have completed a catalog of magnitudes, colors, ellipticities and position angles for 6724 galaxies in the Coma field. Kent and Gunn (AJ 87 , 945) have derived values for projected surface density and velocity dispersion out to a radius of $3^{\circ}$ from the cluster center. Constraints on the anisotropy in Coma are determined; see also Pryor and Geller (ApJ 278, 51).

Luan Ling and Li Jing of the Beljing Astronomical Observatory are analyzing a $9^{\circ} \times 9^{\circ}$ field using $r$ and $J$ UK Schmidt plates to count galaxies to magnitude 20 with a PDS. The globular cluster NGC 1851 is in a corner of the field. They are 
constructing luminosity functions and making statistical analyses of the structure and substructure within clusters.

Bright galaxies with extended halos and sometimes with multiple nuclei are often found in the centers of clusters. Beers and Geller (ApJ 274, 491) show that such galaxies usually lie on significant peaks in cluster galaxy distributions. Despite suggestive circumstances, there are doubts as to whether galactic cannibalism is always taking place (Tonry, ApJ 279, 13; Merrit, ApJ 280, L5). The majority of multiple nucleus systems may only be transcient phenomena. Optical line emission has been found surrounding bright galaxies in the centers of clusters. In addition to M87, Fabian+ (MN 201, 17P) detected gaseous filamentary structure around NGC 4696 in the Centaurus cluster. Van Bruegel+ (ApJ 276, 79) find similar emission around the radio galaxy $4 \mathrm{C} 26.42$ in $\mathrm{A} 1795$. Accretion flows from intracluster gas are thought to be responsible. In some cases, star formation may be a consequence (Fabiant MN 201, 933; Sarazin and 0'Connell, ApJ 268, 552). Materne reports work at the Technical University of Berlin on clusters of galaxies. Studies have been carried out for the Antlia cluster, STR 0627-544 (AA, in press) as well as for Coma and Hydra (AA 13, 85).

At the Befjing Astronomical Observatory, Jia-sheng has derived a power law form of the two-point correlation function, in terms of a natural cut-of $f$ of BBGKY helrarchy equations. The evolutionary history of the correlation function and the effects of peculiar motions and backgrounds are described.

More distant galaxy clusters with substantial look-back times are important for studying the early evolution of galaxies. Efforts have been made to confirm the Butcher-Oemler effect which finds distant clusters of galaxies to contain a high proportion of blue and presumably younger galaxies. The original Butcher-Oemler result was apparently exaggerated in at least one cluster by insufficient correction for foreground and background galaxy contamination (Dressler and Gunn, ApJ 270, 7) and by errors in the photometry (van den Bergh, ApJ 265, 606). Osterbrock (ApJ 280, L43) notes that selection effects can influence conclusions in this type of study. The general consensus seems to be that the effect is real but that careful observations of distant clusters are still needed. Segal (AA 123, 151) deals with the use of clusters as cosmological test objects.

The study of $x$-ray radiation from clusters of galaxies has reached new maturity during the last three years as many of the results from the Einstein Observatory were analysed and published. An excellent review by Forman and Jones appeared (Ann Rev Astron Astrophys 20, 547). Recent work is discussed by Jones and

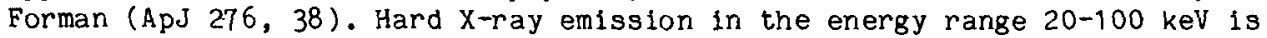
reported in 3 clusters of galaxies by Bazzano+ (ApJ 279, 515). The X-ray luminosity function of Abell clusters at a redshift of 0.25 has been compared with a low-redshift, nearby sample by Henry and Lavery (ApJ 280,1). There seems to be no evidence of any difference between the two distributions, so evolutionary effects must be small. Two-dimensional spectrophotometry of the cores of $x-r a y$ luminous clusters by Cowie+ (ApJ 272, 29) has provided further examples of optical line emission, either from extended 20-100 kpc systems of long filaments or more compact homogeneous regions associated with a dominant central galaxy.

It has long been recognized that head-tail sources found in high resolution continuum maps give unique information about the intracluster gas. Several surveys have been carried out recently (Fantit, AA Suppl 52, 411; Burns and Gregory, ApJ 87, 1245). Einstein X-ray observations for two clusters, A1446 and A1220, which both contain radio tall galaxies are discussed by Burns and Balonek (ApJ 263,546 ). The $\mathrm{X}$-ray data are used to explore mechanisms which may distort radio jets and tails. Slee and Siegman (Proc Astr Soc Aust 5, 114) have used the Culgoora circular array to make 80 and $160 \mathrm{MHz}$ measurements of a selection of cluster radio sources. Owent (AJ 87,1083 ) have surveyed 1478 Abell clusters at $1400 \mathrm{MHz}$ with the NRAO 
$91 \mathrm{~m}$ telescope. 485 sources were found but optical identifications are needed before the data can be fully interpreted.

\title{
4. QUASARS AND RELATED OBJECTS
}

\author{
(M.H. Ulrich)
}

Conferences related to topic have been held: IAU Symposium No. 104 "Early Evolution of the Universe and its Present Structure", G.0. Abell and G. Chincarini editors; IAU Symposium No. 110 "VLBI and Compact Radio Sources", R. Fant1, K. Kellermann and G. Setti editors; XI Texas Symposium on Relativistic Astrophysics, D.S. Evans editor, New York Academy of Sciences, Volume 422; and "Quasars and Gravitational Lenses": $24 \mathrm{th}$ Liège International Astrophysical Colloguium. In general, no references to articles published in the Proceedings of these conferences are made in this Report.

\section{Continuum of the Central Source in Radio, Infrared, Optical, and X-ray}

VLBI observations of large samples of active nuclei have revealed an elongated structure on the milliarcsec scale in a large fraction of nuclei, up to $80 \%$ in the case of quasars (ApJ 269, 387). More cases of superluminal motions have been found including 3C 120, a relatively nearby Seyfert galaxy (ApJ 257, 56). In 3C 345 a small substructure perpendicular to the jet raises the interesting possibility that it could be synchrotron emission from an optically thick disk (ApJ 265, 107). Other evidence for relativistic bulk motions has been provided by the burst observed at $1 \mathrm{~mm}$ in 3C 273 (AA 117, L5). Sensitive searches for radio emission from radio quiet objects have yielded a number of positive detections, suggesting a fairly continuous, rather than bimodal, distribution of the luminosity ratio, $L$ (optical)/L(radio), for active nuclei (except for the BL Lac objects, Kellermannt, preprint).

Infrared/optical observations of high-redshift quasars show that the energy distribution of high and low redshift quasars are similar in their respective rest frame (ApJ 265, 18; ApJ 281, 76) providing circumstantial evidence for the absence of intergalactic dust. Infrared observations of a number of quasars, radio galaxies and Seyferts reveal the fraction of the energy emitted by a non-thermal continuum, a normal stellar population and radiation by dust (AA 107, 276; MN 199, 943 and 953; MN 207, 35; ApJ 252, L53; ApJ 257, L57; ApJ 257, 570; ApJ 272, 400; ApJ Suppl $52,341)$. Some quasars show pure non-thermal IR continuum while some Seyferts produce the IR by dust re-radiation. The $3.3 \mu$ feature which is thought to be a dust indicator is present in IC 4329A and NGC 5506 (AA 100, L16). It has also been reported in the spectrum of 3C 273 and NGC 4151 but it is not confirmed by new observations (Nature 295, 214).

The flux density of NGC 4051 was measured in a ballon experiment at $130 \mu$ and $170 \mu$ (ApJ 274, 571). Preliminary IRAS results show that the strongest far IR emitters are the starburst galaxies and the Seyfert galaxies. The latter have a power-law IR spectrum well represented by $\mathrm{f}_{\alpha} \propto \mathrm{v}^{-1}$, and the far IR flux lies close to the intrapolation of the near IR-ragio spectrum. In contrast, starburst galaxies have a steeper far IR spectrum, $\mathrm{f} \alpha v^{-3}$, and the far IR flux lies well above the intrapolation of the near IR-radio spectrum. Still it is possible that starburst galaxies and Seyfert galaxies are generically related (ApJ 259, 96; AA 124, 89; AJ 88, 1077; ApJ 268,602). Much ef fort has been devoted to the measurement and the interpretation of the "3000 \& bump" in quasars and Seyfert galaxies (ApJ 255,25; ApJ 254, 22; ApJ 257, 487; ApJ 275, 445; ApJ 277, 64).

The $X-r a y$ and $\gamma-r a y$ end of the spectrum is most important in distinguishing among various emission mechanisms, since the bulk of this emission arises close to 
the active nuclei. All active nuclel are strong $X$-ray emitters. Efforts have been devoted to revealing correlations between the luminosity ratios $\mathrm{L}_{X^{\prime}} / \mathrm{L}_{O}$ and $\mathrm{L}_{R} / \mathrm{L}_{\rho}$ and other properties such as the absolute luminosity, the redshift, the mm flux, the radio structure, etc. Among quasars there is a strong correlation between optical and $X-r$ ay luminosities. This correlation is different for radio quiet and radio loud quasars in the sense that for a given optical luminosity the radio quasars are stronger $\mathrm{X}-\mathrm{ray}$ emitters. The region which produces $\mathrm{X}-\mathrm{r}$ ay and optical luminosity in radio quiet quasars may be present also in radio loud quasars, but in addition radio quasars may have a separate source (possibly a jet) emitting in the radio and $X$-ray ranges (MN 198, 921; AA 108, 153; ApJ 260, 437; ApJ 257, 499). The cosmic evolution of the $X-r$ ay properties of quasars and active nuclel has also been investigated (ApJ 268, 60; ApJ 283, 479 and 495). These objects probably contribute $80 \%$ of the $2 \mathrm{keV}$ diffuse X-ray background (ApJ 283,486).

Very rapid variations of the X-ray fluxes have been found in NGC 4051 and NGC 6814 (ApJ 269, L31) although such events seem to be rare among Seyfert galaxies (ApJ 264, 92). Varlations on the same time scales have not been observed in the UV or optical ranges, possibly because the observations have not been conducted with the appropriate time resolution. The spectrum of a number of Seyfert galaxies shows soft $X-r a y$ absorption indicating a higher covering factor of the $X-r a y$ source than in quasars. The absorber has sometimes been identified with the gas producing the broad emission lines (ApJ 262, 564). A sharp X-ray absorption feature has been discovered (ApJ 278, L99) at $600 \mathrm{eV}$ in the BL Lac object PKS 2155-304 but a definite model has not yet been established (ApJ 278, L103). S1multaneous observations of BL Lac objects from $X-r a y$ to the radio range show the overall energy distribution to be consistent with the synchrotron self-compton model in a beamed source (ApJ 253, 19; Advances in UV Astronomy, ed. Y. Kondo, NASA CP-2238, p. 181,185 ; ApJ 253, 38). One week continuous observations of the spectrum of BL Lac show that the time variations of the optical polarization angle and of the total optical flux is consistent with a random walk spectrum (ApJ 260, 415).

POLARIZATION. Several processes, sometimes simultaneously, produce optical polarization in galactic nuclei: intrinsic polarization of the non-thermal continuum, dust, electron scattering by a thin or thick disk. Bright BL Lac objects and violently variable quasars show large intensity varlations of the optical/UV flux, and large variations of the degree and the angle of polarization (MN 198, 1; ApJ 265, 625; ApJ 279, L39). They also have VLBI sources and in those cases where the optical polarization has a preferred angle, the polarization vector is aligned with the VLBI structure (IAU Symposium 110, p. 73 ).

A simple, but not unique, model fitting all these properties is one in which the energy is emitted by a small number of independent non-thermal sources (ApJ 260 , 415), each emitting a fully polarized synchotron radiation of random or tentation and varying on a time scale of a few days. If the emitting material is beamed towards us aberration exaggerates the effects and small changes in the source geometry are amplifled by relativistic effects. Alternatively, if there is no beaming effect then the large intensity outbursts must be due to large increases in the average luminosity of the "independent sources" (ApJ 260, 415). The polarization outbursts would originate from sudden large scale re-orderings of the magnetic field (MN 198, 1).

Most Seyfert 2 galaxies and radio galaxies (ApJ 271,59) show weak polarization with the angle of polarization perpendicular to the radio axis. This suggests the interesting (and speculative) possibility that the polarization is caused by electron scattering in a physically thick disk. Yet, even for well studied objects (NGC 1068, ApJ 271, L7) the interpretation of the observed polarization is ambiguous (Nature 304,609 ). 


\section{The Emission Line Spectrum}

THE BROAD EMISSION LINES. Physical properties of the dense clouds forming the broad line region in Seyfert 1 and quasars are now understood through the detailed analysis of increasingly accurate line intensity ratios (AA 106, 362; MN 200, 271; Astrophys 1cs 17, 1; ApJ 273, 36; ApJ 254, 489; ApJ 260, 44). The discrepancy between observed and calculated intensities of optical multiplets of FeII suggests that energy might be provided to the broad line region under the form of mechanical energy in addition to the radiative energy of the UV/X-ray spectrum (AA 106, 362). Remaining discrepancies between observed and calculated ratios are probably due to the attempt to reproduce an "average quasar" spectrum from well defined physical conditions, while, in fact, line intensity ratios vary from quasar to quasar and, moreover, in a given active nucleus the lines come from different clouds which most likely have different physical conditions.

Two types of data provide clues on the kinematics of the clouds in the broad line regton, the emission line proflles and the change of the line profiles in response to the variations of the central continuum source (see below). Nelther set uniquely constrains the type of motions of the clouds in the broad line region. There is as yet no observational evidence for or against the clouds being gravitationally bound to the central object.

Differences in profiles of emission Iines suggest that not all clouds have the same physical conditions (MN 206, 221; ApJ 259, 48). The symmetry of Lya (MN 201, 645) can be due to pure rotation motions (a not very likely situation) or by emission by optically thin clouds. Additional evidence for both optically thin and optically thick clouds is provided by the difference in redshift between the lines of high and low lonized atoms (ApJ 263,79; MN 207, 73). Finally, it is interesting to note the discovery of two quasars with narrow emission lines (ApJ 252, 69; PASP $95,117)$.

Observations with IUE reveal spectroscopic differences between intrinsically weak Seyfert nuclel and the intrinsically bright quasars: (i) the CIV line has a larger equivalent width (100-200A) in Seyfert galaxies than in quasars $(-30-80 \mathrm{~A})$; (11) line intensity ratios CIV $\lambda$ 1550/Lya and CIV $\lambda 1550 /$ CIII $\lambda 1909$ are both larger in Seyfert nuclei. This suggests a higher degree of ionization in Seyfert galaxies than in quasars and possibly also a higher covering factor of the UV source by the broad line gas in Seyferts (ApJ 266, 28).

The degree of lonization in the broad line region and the absolute intensity of the fonizing flux at $\lambda-1200$ A (measured from the ground in high redshift quasars and with the IUE in Seyfert galaxies) lead to a characteristic linear radius of the broad line region of $-10^{16} \mathrm{~cm}$ to $10^{18} \mathrm{~cm}$. The small linear dimensions of the broad line region in nearby Seyferts (intrinsically weak active nuclei) is confirmed by line variations on time scales of a few weeks found in a number of Seyferts: Akn 120, NGC 3516, NGC 3783, NGC 4151, NGC 4593, NGC 5548, NGC 7469 and others (AJ 88, 926; ApJ 267, 515; MN 202, 455; MN 206, 221; MN 204, 189; Sovlet Astronomy Letters Vol 9; ApJ Supp1 49, 149; AA 132, 136). These observations clearly show the lines varying in response to the continuum variations. In NGC 4151 the varlations can be explained with a time delay of 10 days between variations of the continuum and of the CIV $\lambda 1550$ line, providing, for the first time, a lower limit to the size of the broad line region (MN 206, 221).

THE NARROW EMISSION LINES. Typlcally, these 1 ines have FWHM intensity of $-500 \mathrm{~km} / \mathrm{s}$. Intensity ratios are consistent with photolonization by the central source (AA 135, 341). Classical reddening indicators show that narrow line gas is mixed with dust. Asymmetrical profiles are often interpreted as evidence for radial motions and obscuration (ApJ 281, 525; and M. Whittle 1984, preprint). Evidence for a gradual increase of density and velocity of gas closer to the nucleus is provided 
by the relation between the critical density of a transition and the width of the corresponding emission line (ApJ 275, 493). Lines of very highly lonized species (e.g., Fe IX) also give information on the transition between broad narrow line regions (MN 204, 1231; MN 207, 347; ApJ 273, L31). Improved measurements and analysis of emission line intensities in apparently normal elliptical galaxies show that observed ratios can be produced by a power law spectrum illuminating a gas with a gradient of different densities (AA; ApJ 266, L89) instead of the gas being excited by shocks as previously thought. Intrinsically weak non-thermal continuum sources may be present in nuclei of apparently normal galaxies. The discovery of a Seyfert nucleus in $M 81$ (ApJ 260, 495) suggests that other weak Seyfert line nuclei rema in unknown.

\section{The Absorption Line Spectrum}

THE NARROW ABSORPTION LINES. Spectra of distant quasars show narrow absorption lines at different redshifts which are believed to be caused by gas clouds located along the line-of-sight. Some of these clouds are metal rich and are located in galaxies. Others are detected only in the Lyman lines. These two types of clouds differ by their cosmological evolution, metal abundances, and velocity structure. There appears to be no or only weak cosmological evolution of the number of metal rich absorption IIne systems (MN 198, 91; ApJ 252, 10; Nature 298, 427; ApJ Suppl 48, 455; Quasars and Gravitational Lenses, p. 589; Woolft, 1983, ApJ 268, L1) whereas the number of Lya clouds appears to increase with redshift above what is expected from a uniform space distribution of comoving absorbers. Observations with a resolution of 0.25 A reveal that in Q1101-264 almost all the Lya lines are resolved and have a b-parameter larger than $10 \mathrm{~km} \mathrm{~s}^{-1}$ (ApJ 278, 486). Space Telescope should give the distribution of the absorption system redshifts at $z<$ 1.6. The upper limit to the abundances in the gas producing the "Lya only" absorptions depends on the model for the Lya clouds but it is equal to or below the Population II abundances (ApJ 273, 450; Chaffeet, preprint).

Narrow absorption Iines of CIV 1550 at the redshift of the emission Iines are detected in about half of the quasars. Of particular interest are quasar pairs: the presence in spectra of both quasars of an absorption Iine at the emission redshift of the lower redshift quasar could indicate an extended gaseous halo associated with the lower redshift quasar. However, there exists the alternative possibility that this Iine is caused by a galaxy at the same redshift as the lower redshift quasar (ApJ 261, L7; MN 204, 698; MN 199, 81).

THE BROAD ABSORPTION LINES. Observations of broad absorption line quasars in the optical, $X-r a y$ and radio ranges have been made (ApJ 280, 51; 277, 51; 276, 423; $282,33 ; 280,476)$. Q1414+089 has the broadest absorption 1 ines, with the absorb gas moving relative to the gas producing the emission lines at up to $66000 \mathrm{~km} \mathrm{~s}$ (PASP 95, 341). Models for the formation of the broad absorption 1 ines are discussed (ApJ 265, 73; MN 201, 27).

NEW REDSHIFTS. Quasars, radio galaxies, and Seyferts are discovered through new or continuing surveys (Astrophysics 19, 1; Astrophys 1cs 19, 2; Soviet Astronomy Letters Vol 8; Astron Circ 17; ApJ 269, 73; ApJ 266, 485; MN 200, 191; MN 202, 703; ApJ 266,$485 ; 273,478$ ). Redshifts of several very distant radio galaxies (up to $z=1.819$ for 3C 256; Spinrad, 1984, preprint) have been measured through the intense emission lines which they exhibit (PASP 94, 397).

\section{Extended Nebulosity - Gravitational Lenses}

EXTENDED GASEOUS EMISSION AND UNDERLYING GALAXIES IN QUASARS. An extended envelope of lonized gas has been found around several quasars and radio gaiaxies. In the most extreme examples, MR2251-178 (MN 202, 125) and PKS 2158-380 (MN 201, 991) Ionized gas has been detected up to $170 \mathrm{kpc}$ and $15 \mathrm{kpc}$ from the center, 
respectively ( $\left.\mathrm{H}=50 \mathrm{~km} \mathrm{~s}^{-1} \mathrm{Mpc}^{-1}\right)$. The gas is probably ionized by the non-thermal continuum from the nucleus (see also MN 208, 589 and 955).

Centaurus $A$ is an example of lonized gas detected at large distances from the center. Gaseous filaments $-30 \mathrm{kpc}$ NE of the center are associated with one of the radio lobes and with an extended source of soft $\mathrm{x}$-rays. Optical observations have revealed differential velocities of $\geq 800 \mathrm{~km} \mathrm{~s}^{-1}$ within this group of filaments; they will disperse over a time of $\leq 10^{7}$ years (ApJ 269, 440). An extended region of broad-line high excitation gas has also been detected (Nature 310, p.554; ApJ 273, 154). Additional optical and radio associations are 3C 293 (ApJ 277, 82), 3C 305 (ApJ 262, 529) and NGC 1068 (ApJ 275, 8).

Morphology of Seyfert galaxies has been studied to see how frequent are such pecullarities as condensations and double nuclei (Sovlet Astron Lett Vol 8; Astrofizika Tom 18, 192 and 227; AA 118, 166). Direct imaging of quasars at redshift $\leq 0.6$ shows a surrounding nebulosity with an absolute luminosity and a brightness distribution similar to those of a galaxy (ApJ 262, 48; 257, L1; 278, $11 ; 280,41)$. The nebulosity spectrum is in some cases dominated by a blue continuum and emission lines and in other cases consistent with that of a normal stellar population (ApJ 265, 4; ApJ 265, L1). Quasar flelds have also been imaged in order to see if quasars are more frequently found in tight groups than are average galaxies (ApJ 257, 33; ApJ 271, 471). Evidence has been presented that nuclear emission line activity is more frequent in interacting spirals (ApJ 279 , L5).

GRAVITATIONAL LENSES. In addition to 0957+561A,B and $1115+081 \mathrm{~A}, \mathrm{~B}, \mathrm{C}$, a third case has been reported, $2345+007$ (ApJ 255, L5). Optical and UV observations study the light variations (ApJ 255, 20; Nature 296, 415) and search for deflecting galaxies (Quasars and Gravitational Lenses, p. 182 and p. 192). Images of the triple quasar $1115+081 \mathrm{~A}, \mathrm{~B}, \mathrm{C}$ have been analyzed to find the distribution of mass of the object producing the lensing (MN 200, 941; Quasars and Gravitational Lenses, p. 78 ). Several authors have questioned if all quasars are the result of gravitational lens magnification of a distant Seyfert nucleus by a foreground galaxy (AA 118, L1; IAU Symp 104, p. 375; Quasars and Gravitational Lenses, p. 139 and p. 143). In $2345+007$ several Ly $\alpha$ absorption lines are not in common or are of substantially different strengths. If confirmed this will yleld a size of 5-25 kpc for the characteristic size of the Lya clouds at $z=2$ (ApJ 281, L1).

\section{Models and Evolution}

The black hole and spinar models (ApJ 269, 57) for energy generation have continued to be actively investigated. Spherical accretion (ApJ 267, 18), thin and thick accretion disks have been investigated (MN 203, 323). The key feature of thick disks is their capacity to produce, collimate and accelerate jets (Acta Astr 32, 285; ApJ 266, 502; Astron Gesell Mitterlanger 57, 27). Extraction of energy from the black hole and production of the observed overall continuous spectrum remalns one of the most puzzling and difficult problems (ApJ 267, 371; ApJ 273, L59; ApJ 265, 620; Astrophys Space Sclence 93, 277). Models of quasars as binary black holes (Quasars and Gravitational Lenses, p. 473) or as supermassive stars (Nature 305, 407) have been proposed to explain the broad emission line spectrum. An attractive possibility is that the broad line gas comes from the stars in the nucleus (ApJ 264, 446; ApJ 272, 390).

The search for quasars with $\mathrm{z}>3.5$ (ApJ 253, 28) has produced evidence for an apparent decrease in the space density of quasars at $z>3.5$ (see however: AA 113, 336); samples have increased with the completion of the bright quasar survey. The analysis of these samples leads to the conclusion (ApJ 269,352) that the increase of space density with redshift depends strongly on absolute luminosity, being close to zero for low luminosity quasars. Detailed information about the variation of the 
luminosity function with redshift is still lacking.

\section{The Distribution of Quasars}

A distance criterion unrelated to redshift is to assoclate quasars with other objects of known distance. Reported association of quasars with nearby galaxies (ApJ 271, 479; A+A 138, 408.) have been criticized (MN 200, 47P; ApJ 263, L7); have partially supported (MN 204, 675) or strongly supported associations of quasars with low redshift galaxies (Comptes Rendus 297, 259; ApJ 265, L49 and ApJ 285, 44). One investigation associated quasars with galaxies of similar redshift (ApJ 269 , 29; see also PASP 96,148 ). The subject of alignment, clustering of quasars, and perlodicity in redshifts has received continuing attention (Quasars and Gravitatlonal Lenses, 374; Occas Rep R Obs Edinb 10, 243; MN 201, 179 and ApJ 260, 469; ApJ 277, L27; Journ Astrophys Astron 5, 31; Nature 302, 397; A+A 134, 234; Nachr ichten 305, 339).

\section{GALAXY REDSHIFTS}

(J. P. Huchra)

The last several years have seen both a marked improvement in the instrumentation for small to intermediate diameter telescopes as well as new instrumentation on large telescopes. Photon counting detectors (Shectman and collaborators), CCD based spectrographs (e.g. the "PFUEI" developed by Gunn and collaborators), "Medusae" (fiber optically coupled multiaperture spectrographs; Angel and collaborators), aperture plates (in use at KPNO), plus moveable multislit spectrographs (developed by Huchra and Oke and collaborators) have increased our ability to obtain medium to high quality galaxy redshifts dramatically.

The gathering of high quality redshifts has been spurred on by interest in two major problems: the unraveling of the complexity of the large scale structure in the universe (Early Evolution of the Universe and Its Present Structure, I.A.U. Symposium No. 104); and the structure and evolution of clusters of galaxies (Clusters and Groups of Galaxles, Mardirossian, Giuricin and Mezzetti, Dordrecht, D. Refdel Co.). Rather than slowing down, the growth of the number of redshifts is continuing. The Catalogue of Radial Velocities of Galaxies, compiled by Palumbo, Tanzella-Nitt 1 and Vettolan 1 in 1980 contains 8,250 galaxies with redshifts. The current tally of the CfA Redshift Catalog (Huchra+) is approaching 20,000 galaxies with measidred redshifts. This corresponds to a doubling time of less than five years! Not only is the number of redshifts increasing, so is their quality. $21-\mathrm{cm}$ velocities are routinely measured with errors smaller than $20 \mathrm{~km} / \mathrm{sec}$ and optical velocities (especlally those made with photon counters) also have errors in that range. Older and usually poorer velocities have been replaced and discrepant velocities remeasured.

\section{The Field Galaxies}

Since the last Report, several major field galaxy velocity surveys and catalogs have been published: "Neutral Hydrogen Observations of a Large Sample of Galaxies," Fisher and Tully, 1981, ApJ Suppl. 47, "The CrA Redshift Survey," Huchra, Davis, Latham, and Tonry, 1983, ApJ Suppl. 52 "General Catalog of $21 \mathrm{~cm}$ line Data," Bottinel11, Gouguenheim, and Paturel, 1982, Ast Ap. 106 "A Catalog of HI Measurements of Galaxies, "Huchtmeier, Richter, Bohnenstengel, and Hauschildt, 1983, ESO preprint 250 "A Catalog of Galaxies South of Declination $-30^{\circ}$ That Have Been Observed Spectroscopically," Fairall, Lowe, and Doble, 1983, Pub. of the Dept. Ast. Univ. Cape Town, No. 5. "A Deep Survey of Galaxies," Kirshner, Oemler, Schechter, and Shectman, 1983, AJ 88, 1285. as well as the ESO/Uppsala Survey of the ESO(B) Atlas (Lauberts 1982, ESO, Munich) with many previously unpublished velocities for southern galaxies. These have been supplemented by spectroscopic 
surveys of peculiar galaxies from wide field objective prism surveys (by Markarian and Kazarian, and by Arakelian, Petrossian, Lipovetski i and collaborators at Byurakan and at the Special Astrophysical Observatory $(6 \mathrm{~m})$ and published in a series of papers in Astrofizica).

Large bodies of unpublished redshifts are associated with new and/or continuing surveys. Glovanelli and Haynes are continuing their HI measurements of galaxies from Arecibo. The Center for Astrophysics survey is being pushed to a magnitude Iimit of $B=15.5$ (above Dec $=0$ and $b>40^{\circ}$ or $b\left\langle-30^{\circ}\right.$ ). When completed, this will be a magnitude limited survey that will reach a mean almost $10,000 \mathrm{~km} / \mathrm{sec}$ and contain over 8000 galaxies. Kirshnert are continuing to survey the Void in Bootes. The analogue of the original CfA survey (magni tude 1imit of approximately $B=14.5$ ) is now underway in the Southern Hemisphere with several groups (Da Costa and collaborators in Brazll, W.L.W. Sargent and collaborators at Las Campanas and also SAAO, and Fairall at SAAO) surveying different areas of the sky.

Several groups are also surveying very small regions to faint limiting magnitudes (Ellis and collaborators, Kron and Koo, and Geller and collaborators). A few groups are also pursuing the use of objective prism surveys (Palmer 1983 in Clusters and Groups of Galaxies) and galaxy colors (KoO+ in the same volume; Loh, June 1984 A.A.S. meeting) for large scale surveys, but the best redshifts available from the prism techniques are only $+/-3000 \mathrm{~km} / \mathrm{sec}$, while multicolor work seems to be reliable only for studying clusters of galaxies.

\section{Binarles and Groups of Galaxies}

An exhaustive reanalysis of the dynamics of Turner's binary galaxies was done by White+ (1983, MN 203, 701). With significantly better velocities they found that mean $\mathrm{M} / \mathrm{L}$ is approximately 50 to $100 \mathrm{~h}$ (in solar units), essentially the same answer as Turner. Tifft has continued his investigations of double galaxies (ApJ 257, 442; ApJ 262, 44; ApJ 268, 56; and following papers) and cont inues to find evidence for the quantization of redshifts. This effect is neither well understood nor generally accepted although the velocity measurements are of very high quality. Karachentsev and Karachentseva are also continuing their studies of interacting double and triple galaxies.

Two new major compllations of galaxy groups have been published. Hickson and collaborators are studying a sample of compact groups of galaxies (1982, ApJ 255, 382; and in Clusters and Groups of Galaxies). Geller and Huchra have identifled groups with complete dynamical information from the Revised Shapley Ames Catalog (Sandage and Tammann, 1981 Carnegie Institution, Washington) and also from the Cf A Redshift Survey (Huchra and Geller 1982, ApJ 257, 423; Geller and Huchra 1983, ApJ Supp1. 52,61). A summary of this work can be found in Clusters and Groups of Galaxies. Theoretical work on the interpretation of group and cluster positional and velocity information is avallable from Bahcall and Tremaine and collaborators (Bahcall and Tremaine 1981, Ap. J. 244, 805) and Mezetti, Mardirossian and Giuricin (1983, AA 125,368 ; AA 126,86$)$.

\section{Clusters of Galaxies}

Interest in the internal dynamics of clusters of galaxies has grown with the advent of powerful $\mathrm{X}$-ray telescopes in space (Einstein and HEAO-A) and with improved dynamical and evolutionary models for clusters. "Deep" surveys of clusters have revealed that these objects are physically very complex (Beers+ 1982, ApJ 257, 23; Bothun+ 1983, ApJ 268, 540; Huchra+ 1983, in Clusters and Croups of Galaxies). Extensive velocity surveys of most nearby northern cluster of galaxies are nearing completion as part of the CfA Redshift survey. More distant clusters and southern clusters are now being surveyed by several groups: Chincarini and collaborators 
(Hercules and Perseus), Melnick and Quintana (southern Hemisphere), Dressler and Shectman (selected clusters) and Geller and Huchra and collaborators (CfA overlap Pegasus, Cancer, Virgo, Coma, A1367).

Significant surveys published in the last two years include the Coma Survey of Kent and Gunn (1982, AJ 87, 945), the Hydra I survey of Richter+ (1982, AA 111, 193), the Perseus survey of Kent and Sargent (1983, AJ 88, 489). Huchra has over 600 velocities for galaxies in the core of the Virgo cluster (1985 ApJ in press). It is also in the study of medium distant galaxy clusters that multi-object spectroscopy can really come to the fore (e.g. Hintzen+ 1982, AJ 87, 1656).

Spinrad and Longair and collaborators have continued the use of radio sources to identify high redshift galaxies (usually brightest cluster members). There are now more than 25 galaxies (clusters) with measured redshifts greater than $z=0.75$, with the largest galaxy emission line redshift at $z=1.819$. Several clusters with $z>0.15$ have even had their velocity dispersions measured (Abell 115: Beers+ 1983, ApJ 264, 356; 3C295, C10024+1654: Dressler and Gunn 1983, ApJ 270, 7; preprint).

\title{
IV. Superclustering
}

Studies in the large scale structure of the universe have increasingly used clusters of galaxies as tracers of that structure. Oort has compiled an excellent review of earlier work (1983, Ann. Rev. 21, 373). Over 600 redshifts are now available for Abell clusters (Postman+ 1985, ApJ in press). Several groups are working on surveys of cluster velocities including Karachentsev and Kopylor in the Soviet Union, Burns and Batuski at Kitt Peak, Ford and Harms and collaborators at Lick and Kitt Peak (1983, ApJ 273, 24), Shectman at Las Campanas and Palomar and Huchra and Henry at Mt. Hopkins and Hawali.

Nearby superclusters (including our own) have also been extensively studied as well as Coma, Perseus, Hercules and the newly uncovered Lynx-Ursa Major chain (Giovanelli and Haynes 1982, AJ 87, 1355). According to some authors, the magnitude of the infall of the Local Group into Virgo is approximately $250 \mathrm{~km} / \mathrm{sec}$ (Aaronson+ 1982, Ap. J. 258, 64). A next major step will be more detailed mapping of the distortion in the velocity field caused by the flattening and non-uniformity of the Local Supercluster mass distribution.

\section{EXTRAGALACTIC RESEARCH IN THE U.S.S.R}

(I.D. Karachentsev)

\author{
Af - Astrofizika, Erevan \\ $A Z$ - Astron. Zhurnal \\ LAZ- Letters to AZ (Pis'ma) \\ AC - Astron. Circ. \\ Crim - Izvestia Crimean Astrophys. Obs. \\ LGU - Trudy Astron. Obs., Leningrad Univ. \\ AfI - Trudy Astrophys. Inst., Alma-Ata \\ Tartu - Publ. Tartu Obs. \\ DArm - Doklady Acad. Nauk Armenla \\ Kazan - Trudy Kazan Astron. Obs. BAO - Communic. Byurakan Astrophys. Obs. \\ MGU- Trudy Sternberg State Astron. Inst., Moscow
}

\section{Surveys and Lists of Galaxies}

Markarian and Stepanian (Af 19,639; 20, 21) began a new survey of objects with UV-excess on the Byurakan 40" Schmidt telescope with an objective prism. Using combinations of three prisms and IIIaF, IIIaJ emulsions, they reached the survey limiting magnitude -19.5 . In two areas $0950+55$ and $1130+59,40 \times 40$ each, they have found 252 blue objects. The relative number of QSO and Seyfert galaxles among them is expected to be $-20 \%$. Kazarian and Kazarian (Af 18, 512; 19, 213) have presented a list of 228 blue galaxies which were found at the same telescope with a $1: 5$ prism applying a softer UV-excess criterion. 
Abdulova and Denisjuk (AfI 40, 41) have compiled a catalogue of radial velocities for 408 emission galaxies observed on the $0.7-\mathrm{m}$ telescope with an image tube. For -400 interacting galaxies a reference list of radial velocities is prepared by Vorontsov-Velyaminov and Arkhipova (MGU 53, 3). Redshifts for 130 faint blue galaxies located in the Virgo cluster center direction were published by Karachentsev + (LAZ 8, 198; 9, 387). Kost juk and Kopy lov (LAZ 8, 520) measured radial velocities for 53 ring-like galaxies from Kostjuk's list. "Catalogue of 603 isolated pairs of galaxles" by Karachentsev is now recorded on magnetic tape. The initial version of the catalogue is supplemented by new data on radial velocities, spectral types and orbital masses for all double galaxies. Kogoshvili (Af 19, 584) studied the inhomogeneity of the data in the Morphologic Catalogue of Galaxies by Vorontsov-Velyaminov.

\section{Active Galactic Nuclei}

Markariant (Af 19, 221; 20, 213; AC 1233, 1) and Denisjuk+ (Af 19, 229; 18, 329 ) continued spectral observations of Markarian galaxies from Byurakan surveys. Slit spectra have been obtained by Kazarian and Tamazian (Af 18, 192, ; LAZ 8, 454) and Kazarian (Af 19, 411; 20, 35; LAZ 9, 648) for many galaxies having UV-excess. Markarian galaxies, especially with double-nuclei structure, have been studied spectrally by Petrosian (AC 1231,$3 ; 1266,1 ; 1277,2$ ) and Khachikiant (Af 19, 239; $19,393, ;$ LAZ 8,270$)$. Spectral observations and emission line profile classification have been made for 38 Seyfert galaxies by Doroshenko and Terebizh (MGU 55, 64), Doroshenko+ (Af 19, 823) and Afanasjev+ (Af 18, 329). Velocity fields in the central regions of Mkn 538 and Mkn 617 have been investigated by Afanasjev and Rassokhin (LAZ 8, 515; 9, 389). Morphological properties of Markarian galaxies have been considered by Petrosiant (Af 19,619; 171; 20, 51), Petrosian (Af 18, 548; LAZ 9, 339; AC 1277, 5) and Eghiazariant (Af 19,631; 18, 541). Integral characteristics of superassociations and of galaxies with double nuclei are presented.

The UBVR observations of galaxies with active nuclei have been continued. Doroshenko and Terebizh (Af 19,5) have investigated 22 Seyfert galaxies in the UBVR system and in the narrow band filters. Dibay+ (AZ 61,1) have measured $\mathrm{U}, \mathrm{B}, \mathrm{V}, \mathrm{R}$-magnitudes for 37 Markarian and Arakelian galaxies. Scanning 17 Seyfert and 7 normal galaxies with the slit spectrophotometer Afanasjev+ (LAZ 8, 579,; SAO 33, 5) have confirmed the interconnection of Seyfert properties with a volume luminosity at their circumnuclear regions. Photometry of a number of UV-excess galaxies has been made by Tamazlan (BAO 54, 80; Af 20, 43), Kalloglian and Börngen (Af 19, 403) and Hagen-Torn+ (Af 19, 199). Optical variability has been detected in Seyfert galaxies Mkn 699 (BAO 58, 99) and IV ZW29 (LAZ 9, 82). Pronik studied the emission line varlablity in NGC 1275 and NGC 3227 (Af 19, 245; AC 1209, 1; Crim 68, 93).

Radio observations of -400 active nuclel galaxies from Byurakan lists have been made at $327 \mathrm{MHz}, 102 \mathrm{MHz}$, and from 3.9 to $31 \mathrm{~cm}$ by Sanamiant (Af 18, 651; 19, 429 ) and by Artyukh+ (Af 18, 215; LAZ 9, 86). Other such objects were also studied (AP 18, 343; AC 1247, 1). The radio-luminosity function of Seyfert galaxies has been discussed by Kandalian (Af 18, 580). A dependence of flux and radio-spectrum of a galaxy on the type of its nucleus according to Byurakan classification was noted (Af 19, 251; 18, 25; 19,655). Physical conditions in the nuclei of Seyfert galaxies and the nature of their activity have been considered by Dibay+ ( $\mathrm{AZ}$ 60,$441 ; 61,209 ; 61,10 ;$ AC 1216,1; 1269,1; LAZ 9,707), Zentsova (AZ 59,1089; 60,34; 846 ; 1081; LAZ 8,535), Komberg (AZ 59,1062) and by others (Af 19,15; AfI 39,21; $39,83$; LAZ 8,263; AC 1264,1$)$. 


\section{Compact Galaxies and Quasars}

Börngen and Kalloglian (Af 18, 17) have measured the brightness distribution for 262 compact galaxies. Arkhipova and Savel'eva (MGU 54, 33) have made photoelectric UBV observations for 49 compact and 70 interacting galaxies. The spectrophotometry of 4 Arakelian galaxles is presented (LAZ 8, 334). Hagen-Torn+ (LGU 410, 104; 413,43) continued photometry of compact extragalactic objects. Malymian+ (Af 18, 324; AZ 59, 1075) have carried out a survey of Arakelian galaxies, having high surface brightness, at frequencies of $3.66 \mathrm{GHz}$ and $102 \mathrm{MHz}$.

In the Byurakan survey, Markar lant (Af 19, 29; AC 1233, 2; 1265, 1) have found 37 new quasars. Spectral and photometric observations of $O Q 172$ and $1136+486$ are reported (LAZ 10, 83; AC 1241, 1). A search for optical varlability was provided (Af 18,657; 18, 245). Artyukh (AZ 61, 240) has observed quasars from Parkes survey at $102 \mathrm{MHz}$. Bochkarev and Antokhin (AC 1238, 1; AZ 60, 448) have proposed an interpretation of a possible variability of emission lines of quasars. Komberg and Sheffer (LAZ 9, 529), Katysheva (Af 19, 55; AZ 61,35), and Grinin (AZ 59, 1070) investigated energetics and dynamics of emitting gas in quasars. Levshakov and Varshalovich (Comments Astrophys 9, 199; Af 18, 49), Waltz and Komberg (AZ 59, 844; Af 19,449$)$ have undertaken new attempts to identify absorption lines in quasar spectra. Evolution changes in the number of absorption 1 ines for quasars has been estimated by Nazarov (AC 1284, 1). Komberg (Af 20,73) has assumed the existence of two populations of quasi stellar objects. Lipunov and Sazhin (LAZ 8, 649) connect quasar activity phenomena with gravitational waves. QSO Iuminosity evolution was considered (AZ 60, 840).

\section{Extragalactic Radio Sources}

With the RATAN-600, Amirkhanlan+ (SAO 36, 50) have made a sky survey within the declination range $4^{\circ} 00^{\prime}$ to $5^{\circ} 50^{\prime}$ at $3.9 \mathrm{GHz}$. In a total area of 0.17 steradian there have been found 585 radio sources, including 329 new ones. Us ing the same radio telescope, Malymian (LAZ 10,90) observed 15 radio galaxies from Bologna survey, and Ohanian, Panajian (BAO 54, 10) have measured fluxes and spectral indices at $968 \mathrm{MHz}$ and $3.66 \mathrm{GHz}$ for 20 objects from UTI-1ists, drawn up from lunar occultations. Artyukh+ (BAO 54, 3) have determined angular dimensions of 52 UTI-sources, using interplanetary scintillations at $102 \mathrm{MHz}$.

Efanovt (Crim 66, 205) have obtained high-frequency spectra for 143 rad10 sources and estimated their optical depth at 8-22 $\mathrm{GHz}$. observing 8 variable sources, Efanovt (Crim 68, 104) have shown that the most intens ive flux variations occur at $\lambda=(1.3-2.8) \mathrm{cm}$. Sobolevat (LAZ 8, 205; 10, 585) discovered extended

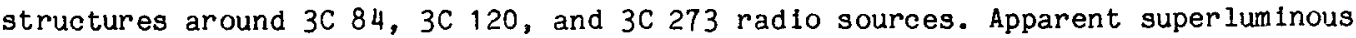
motions have been analyzed by Kyril'chik ( $A Z 59,657$ ) in the fine structure components of $3 \mathrm{C} 120$.

\section{Structure and Kinematics of Galaxies}

An extensive investigation of the system of globular clusters in $M 31$ was undertaken by Sharov and Ljuty (AZ 60, 1;666;61,245) and by Sharov+ (LAZ 8, 451; 9, 393). UBV magnitudes are measured for several hundred clusters at the $2.6 \mathrm{~m}$ and $6 \mathrm{~m}$ telescopes. Conclusions based on modern data are presented in Sharov's monograph "Andromeda Nebulae" (Nauka, Moscow, 1982). Efremov (LAZ 8, 339; 585) studied stellar complexes in M 31. Efremov and Ivanov (Astrophys Space Sc1 86, 117) have found a relation between a volume density of the brightest stars in an $M 31$ arm and a gradient of age for these stars.

Karachenstsev and Mineva (LAZ 10, 253) measured rotation curves, $V(R)$, for 21 isolated galaxies and for 37 double galaxies (LAZ 10,563), and obtain the mean mass-to-luminosity ratio $7 \mathrm{M}_{\odot} / \mathrm{L}_{\odot}$. Zasov and Kyazymov (AZ 60, 656) have complled a 
list of -100 normal galaxies with $V(R)$ determination out to large $R$. They noted that Keplerian rotation curves are relatively rare $(-20 \%)$ and usually occur among galaxies having close neighbours. Determinations of $V(R)$ for the interacting galaxies VV48, VV219, and for the irregular galaxy NGC 2814 have been made (AZ 60, 20; Af 18, 337). Spectral and photometric investigations of NGC 2685, 2184, 2968, 3034 , and 3718 are presented (Af 19, 599; 19, 45; 19,575; 18, 31; LGU 413, 73). Zasov (AC 1232,1) considered a galaxy bulge influence on its rotation curve properties.

Pasha+ (Astrophys Space Se1 86, 215; MGU 53, 15) and Korchagin (AC 1221, 1) continue the study of regularities of spiral patterns. Nazarova (Kazan 47,$66 ; 48$, 47) investigated the distribution of hot spots in the central parts of NGC 2903, 3310 , and 6951. Iskudar ian (DArm 74, 122; 217), Vorontsov-Velyaminov (AC 1257, 7), and Fessenko (Af 18, 37) consldered some relations between integral properties of galaxies of different structural types. A SN burst frequency was estimated for spiral galaxles (Af 18,563; LAZ 9, 478; AZ 61, 234).

Sil'chenko with Zasov and Tarasova (AZ 59, 855;60, 1063;61, 45; LAZ 10, 19) have performed evolution simulations of colors and spectra of galaxies, and also of M/L for different type galaxies. These calculations define the star formation intensity using its integral properties. Estimates of the temperature of gas sweepling out of a galaxy due to radlative pressure from active nuclei, and also as a result of galaxy motion through intergalactic medium, have been made by Corbatsky (Af 18, 234) and Kritsyk (Af 19, 471).

\section{Systems of Galaxies}

Karachentsev (LAZ 9,67) has finished radial velocity measurements for the double galaxies in $\mathrm{h}$ is catalogue. Individual masses have been determined for 227 members of pairs. A joint analysis of orbital and individual masses confirms two main conclusions: galaxy orbital motions are nearly circular; and the essential part of the mass of members of the pairs is concentrated within their standard radii $R_{25}$. Karachentsev and Shcherbanovsky ( $L A Z 9,332$ ) have investigated the double galaxy centers distribution and found considerable difference of the two-point correlation function, $\xi(r)$, for them from that for galaxies. Arakelian (Af 19, 666; 673) and Petrosian (AZ 61, 441) studied a luminosity function for isolated pairs of galaxies and the role of different selection factors. Dostal (Af 18,201 ) and Tovmass ian (Af 18, 227) discussed observable manifestations of galactic nuclear activity and of interaction phenomena in tight double systems. Bysnovaty-Kogan (LAZ 10, 181) analyzed rotation of a galaxy in a pair taking into account tidal forces.

Karachentseva and Karachentse $v$ continued radial velocity measurements for triple system members. Virial mass estimates have been made for 48 triplets of galaxies (Af 18, 5; 19,613). Vennikt (Af 18,523; 533) measured redshifts for 110 galaxies in the vicinity of nearby groups. Karachentsevat (AN 303, 189; 305, 53; 305 , 59) have discovered many new dwarf members of M 81 group, resolved into stars. Typical luminosity profiles follow the exponential law. Arkhipova (AZ 59, 209) made UBV photometry of 80 "nests" of interacting galaxies. Kuz'min ( Tartu 49, 140) and Jaaniste (Tartu 49, 170) studied orientation of galaxies in groups. The statistics of structural types and of nuclear classes of galaxies in groups have been considered by Magtesian (BAO 53, 102).

Shcherbanovsky (SAO 31, 21) and Joeveer (AC 1239, 1) investigated the space structure of Virgo cluster of galaxies. The virial mass of this cluster and its luminosity function down to $\mathrm{m}=12^{\mathrm{m}}$ have been determined by Karachentsev (Af 18 , 501). Zasov ( LAZ 9, 327) has supposed that Virgo cluster has an extended envelope populated by blue dwarf galaxles. Kallogliant (Af 19, 183) have made two-color photometry of the cluster A 1185. Gubanov (Af 18, 177; AZ 60, 1051) and 
Dagkesamansky+ (MN 200, 971; AZ 60,9) have made radio observations of Abell clusters at $102 \mathrm{MHz}$. Interaction of intergalactic gas with cluster galaxies was studied by Syunjaev (LAZ 8, 323), Gorbatsky (Af 20,61), Volkov and Ivanov (Af 19, 91). Sholomitsky (LAZ 10, 260) considered the possibility of the submillimeter spectroscopy to measure peculiar velocities of clusters of galaxies.

\section{Large Scale Structure of the Universe}

Joeveer (Tartu 49, 161) studied the space structure of the Local Supercluster. Einasto+ (Highlights Astron 6,757, Patras) have presented evidence for existence of two local giant voids with the diameter about $200 \mathrm{Mpc}$. Us ing the radial velocities of 6775 galaxies with $V<17000 \mathrm{~km} / \mathrm{s}$, Einastot (MN 206, 529) investigated the Local Supercluster geometry. Applying methods of correlation functions, cluster analysis, and percolation theory, they found that $95 \%$ of the galaxies belong to the filament system, which occupy only $5 \%$ of the volume under consideration.

Tago+ (MN 206, 559) have studied the space distribution of Zwicky clusters in the wide vicinity of the Coma supercluster. They detected chain-like structures at $80 \mathrm{Mpc}$, which connect Coma with the Local supercluster and Hercules supercluster. Gubermant (Af 19, 97) have made a geometric analys is of different catalogues of galaxies to verify the consequences of the "pancake" theory of galaxy system formation. Pavlova (AfI 40, 34) studied the or lentation of galaxies in Perseus and Pisces regions. Fessenko ( $L A Z$ 8, 458; Af 17, 719; AZ 60, 1077) has analyzed different effects of observable biases, which play an important role in selection of superclusters of galaxies. The space correlation function for rich clusters of galaxies were derived by Klypin and Kopylov (LAZ 9, 75) on the scale of 5 to 100 Mpc. Its amplitude exceeds that for galaxies. According to Karachentsev (LAZ 10, 175), the correlation function for blue emission galaxies has negative amplitude with in the range 30 to $60 \mathrm{Mpc}$ and a secondary peak at $-90 \mathrm{Mpc}$.

\section{WORKING GROUP ON THE MAGELLANIC CLOUDS \\ (M.W. Feast)}

A complete bibliography of $M C$ research is found in the special section on the $M C$ in $A$ and A Abstracts. An overview of much current research on the $M C$ is given in the volume of IAU Symposium 108 (Structure and Evolution of the Magellanic Clouds = S108). Both IAU Symposium 105 (Observational Tests of Stellar Evolution Theory) and IAU Colloquium 82 (Cepheids, Observation and Theory $=$ C82) contain much important MC data.

\section{30 Doradus, HII Regions, Interstellar Extinction}

In 1981 it was suggested that R136, the central object of the 30 Doradus HII region was a supermassive object $\left(-3000 M_{\odot}\right)$. The arguments involved the high brightness of the object (if single), the supposed uniqueness of the ultraviolet spectrum and the possible light variability. Many papers have subsequently appeared (S108). It now appears that the ultraviolet line spectrum is consistent with the presence of one or more 03 stars and that evidence for variability is dubious (ApJ $273,597)$. Moreover the object has been split into a number of components. The most detailed work is that of Walker and $O^{\prime}$ Donoghue (Ast Exp, in press). The upper limit to the mass of the brightest component is 200-300 M and this component may be a small group of normal stars. The mass loss rate of $\$ 136$ (AA 120,269) and the infrared excess (ApJ 272, 123) probably need reassessing in the light of these results.

McGregor and Hyland (ApJ 250, 116) use JHK photometry to deduce that there has been at least two bursts of star formation in 30 Dor during the last $5 \times 10^{7}$ years. 
Evidence for metal deficlency in 30 Dor supergiants is also given. Work on WR stars in 30 Dor is discussed in IAU Symposium 99. Further studies have been carried out on the large scale gas motions in 30 Dor (AA 117, 265; MN 196, 19p). The halo of 30 Dor consists of expanding lonized shells. $1410 \mathrm{MHz}$ observations (AA 133, 93) reveal a low density component extending over 2100pc. Huchrat (ApJ 274, 125) discuss star formation in 30 Dor in relation to that in blue galaxies. Caplan and Deharveng (S108, AA and AA Suppl submitted) have made absolute $H \alpha, H \beta$ photometry of 75 LMC and SMC HII regions. The data yield reddenings and indicate dust within the HII regions. Regions of high excitation have been found in LMC N11 and N159A (AA 118, 116; 111, L11). ${ }^{12} \mathrm{CO}(\mathrm{J}=2-1)$ has been detected in three LMC HII regions (ApJ 262, 100). The SMC appears to be significantly deficient in CO with respect to the Galaxy whilst the LMC is at most marginally deficient. In MC HII regions the observed depletion of carbon is consistent with most of this element being produced in stars in the mass range 4-10 $\mathrm{M}$ (ApJ 252, 461). The frequency distribution of HII region dlameters in the MC is di scussed by van den Bergh (AJ 86,1464 ). The S/0 abundance ratio in MC HII regions and planetaries is the same as in our Galaxy (ApJ 244, 54; AA 118, 234). Other abundances and temperatures are discussed by Mathis (ApJ 261, 195). Lequeux+ (AA 103, 305) and Hunter (ApJ 260, 81) relate the properties of MC HII regions to the history of star formation in these regions. The velocity fleld of supergiant shell LMC 2 shows that it may result from combined action of stellar winds and supernova explosions (AA 110, 185). Line ratios and velocities have been derived for a number of giant LMC bubbles. New velocity components in several giant interstellar shelis have been detected (MN 206, 705; ApJ 255, 447). Theoretical arguments suggest that giant LMC gas clouds are unstable (ApJ 252, 481).

The $M C$ is a fruitful region for the study of very early stages of star formation. $\mathrm{H}_{2} \mathrm{O}$ masering from a number of MC HII regions has been reported (AJ 87 , 528; MN 205, 275) and although the one in N159 (LMC) was not detected at a later date (MN 200, 19p) both this region and N105 (LMC) (MN 197, 23p) show type I OH masering. Infrared work revealed a very red object in N159 which is believed to be similar to galactic infrared protostars (MN 197, 17p).

Extensive work on the nature of the interstellar particles in the MC has continued. In both clouds the far uv extinction law differs from that in our Galaxy wi th the $2200 \mathrm{~A}$ feature being weak or absent in MC. The effect, more pronounced in the SMC than LMC, appears associated with a deficiency of graphite grains in MC (cf. ApJ 247,860; 255,70, S108; AA 132,389; MN 201, 1p; 204, 29p). Nevertheless some SMC stars (particularly SK 143) have a normal galactic type uv extinction (AA 113, L15, S108). The gas/dust ratio for this star is similar to typical galactic values whereas for most MC stars it is much higher. Furthermore, some galactic objects (the Plelades star HD23512, ApJ 244, 199) have a weak 2200A feature as in MC stars. The LMC extinction law has been put in analytical form (MN 203, 301). Nandy+ find that the strength of the diffuse band at 4430A in LMC stars is not related to the population of small grains responsible for the far uv extinction (Ast Sp Sc 85, 159). Fortunately for the practical problem of making absorption corrections, infrared photometry has been used to show that in both clouds the ratio $R=A / E_{B-V}$ is close to the galactic value (3.05) (MN 199, 979; AA 107, 247; Obs 104, 193). B Accurate BVI reddening for 77 MC Cepheids give mean values of $E_{B-V}=$ 0.074 (LMC) and 0.054 (SMC) each with small dispersion (Caldwell and Coulson, MN, in press). The wavelength dependence of interstellar polarization $(0.36-0.84 \mu \mathrm{m})$ in the LMC fits the Serkowski galactic empirical relation well and a similar range of $\lambda$ (max. polarization) is found (ApJ 265, 194).

\section{Supernova Remnants, X-ray Sources and Planetary Nebulae}

The MC have proved exceptionally important in the study of SNR's (IAU Symposium 101 and S108). Remnants have been identified from optical, radio and $X$-ray surveys (ApJ Sup 51,$345 ; 55,189 ; \mathrm{MN} 200,1007$ ). The ir frequencies are 
estimated as one in -275 years (LMC) and -800 years (SMC). The first remnants of SNI outside our Galaxy have been identifled in the LMC (ApJ 261, 473) and the first oxygen-rich, young SNR in the SMC has been found (ApJ 248, L105). X-ray spectra of 4 LMC SNR are consistent wi th thermal emission whilst two others are probably of synchrotron origin (ApJ 255, 440). Detailed study of N63A and N49 show these SNR's to be evolving in a heterogeneous, cloudy interstellar medium (ApJ 275, 592, 611). 30 Dor $B$ has radio and X-ray properties similar to the Crab Nebula (MN 202, 927). Absolute photometry of several SNR confirms the model of optical emission originating in dense cloudlets embedded in a tenuous medium (AA 111, 171). N185 (LMC) probably originated in an SN explosion (AA 115, 61) whilst N70 (LMC) is a mass loss bubble within a massive collapsing HI Cloud (ApJ 250, 103). The gamma ray burst from the LMC SNR N49 may have been due to the nonexplosive collapse of a white dwarf which exceeded its mass limit (ApJ 261, L71).

Individual binary $X-r a y$ sources in the MC are discussed by Commission 42 and in S108. A significant result is the identification of LMC $X-3$ as a black hole candidate (ApJ 272, 118; 273, L81; 274, L65; AA 126, 265; 132, 240). The Einstein satellite has detected $75 \mathrm{X}-\mathrm{r}$ ay sources in the LMC, concentrated around 30 Dor. At least 25 of the sources are SNRs (ApJ 248, 925).

Work on MC planetaries is discussed in S108 and in IAU Symposium 103. Carbon abundance is similar to that in galactic planetaries, in contrast to the MC interstellar medium in which carbon is very under-abundant (ApJ 253, L43). Further studies of abundances in LMC PN show (ApJ 273,590) He, $\mathrm{N}$ and 0 varying with object, but with $\mathrm{Ne}$ and Ar remarkably constant. IUE observations of the central stars of 3 MC PN's lead to M-1M, L-4.5 $\mathrm{M}_{\odot} 10 \mathrm{~L}$ and $\mathrm{T}-1 \times 10^{5} \mathrm{~K}$. The progenitors were probably $4 \mathrm{M}$ stars and the upper limit to the PN nuclei luminosities gives upper 11 mits to the MC distances (ApJ 262, L41). Planetary Nebula formation in various galaxies including the $M C$ is discussed by Papp+ (ApJ 268, 145). New results on excitation classes of MC PNs confirm earlier work (MN 208,633).

\section{Cepheids, RR Lyrae Stars, Blue Supergiant Variables, Novae}

Only work subsequent to $\$ 108$ and $\mathrm{C} 82$ are referenced here. The Cape photoelectric BVRI photometry of SMC Cepheids (SAAO Circ 8, 1) and the Dunsink photographic observations of LMC Cepheids (AA Sup 56, 169) are now published. Extensive radial velocity work on MC Cepheids using CORAVEL is in progress. Observations of long perlod "pseudocepheids" is reported by Eggen (AJ 88, 1458). The first accurate magnitudes and colours of MC RR Lyrae varlables have been obtained by Walker (MN, in press). He observed the LMC cluster NGC 2210 with a CCD camera. If $\left\langle M_{\mathrm{v}}(\mathrm{RR})\right\rangle=0.6$ then $(\mathrm{m}-\mathrm{M})=18.42 \pm 0.10$. Graham has found additional RR Lyrae variables in this cluster ( $\left.\beta_{A S P} 93,546\right)$.

Work on S Dor variables and related objects has continued both because of their importance for understanding stellar evolution in massive stars and as possible distance indicators (AA 127, 49; 110, 272; 112, 61; ApJ 273, 177; IAU Symposium 98). Some but not all $S$ Dor variables show circumstellar dust emission (MN 209, 759; AA 120, 287; 131, L5). Amongst suggested mechanisms for these objects is sudden massive outflow of matter possibly coupled with radial oscillations of the stellar envelope (ApJ 264, 583). A remarkable -1 mag outburst of R127 was recorded (IAU CIrC 3767).

Data on novae in the MC were reviewed at S108. No new novae were reported during the period under revlew: the importance of a continuing survey for MC novae needs stressing. Photometric and spectroscopic observations of Nova LMC 1981 were reported (AA 124, 143; IAU CIrc 3648) and the spectrum of Nova LMC 1977b was described (PASP 93, 581). An eruptive variable in the LMC was reported (IAU Circ 3662). 


\section{Red Stars (including variables)}

The study of red stars continues to be very active ( 108 and IAU Symposium 105; review by Catchpole and Feast, Strasbourg meeting on Cool stars with excesses of heavy elements, in press). Spectra and infrared photometry of red giants in MC clusters (MN 202, 59) are particularly important in understanding the evolutionary connections of $M, C$ and $S$ type stars. In intermediate age clusters $C$ stars first appear on the $A G B$ at $M=-4.3$ to -4.6 (though there is a separate fainter class of ${ }^{13} \mathrm{C}-\mathrm{r}$ ich $\mathrm{C}$ stars). Bgservations in clusters confirm the association of $\mathrm{S}$ stars with helium shell-flashing in a transition phase from $M$ to $C$ types. The $M$ to $S$ and $S$ to $C$ transitions occur at higher luminosity and lower surface temperature in the younger (more massive) stars (MN 204, 985). IR photometry and vi sual spectrophotometry has been compared with theory to deduce the evolutionary history of $M$ and $C$ stars in MC clusters and in the general field (ApJ 253, 580). Differences between $M$ star populations of LMC and SMC clusters is attributed to age and/or metallicity effects. The ef fect of $C$ stars on the integrated light of MC clusters was studied (ApJ 266, 105) on the basis of extensive infrared photometry. Lack of high luminosity $C$ stars is notable. Integrated infrared colours of clusters were analysed as a function of cluster age. Use of infrared luminosities of $M$ supergiants as extragalactic distance indicators was suggested (ApJ 249, L55). The high proportion of $C$ to $M$ giants in the SMC compared to the Galaxy has now been found to extend to the cool components of symbiotic systems in the two galaxies (MN 203, 25; see also ApJ 275, 584). New Mira variables in the LMC have been discovered by Wood, Bessell and Paltoglou (ApJ, in press) and by Reid and Glass (to be published). These show a well defined period-luminosity relation with carbon and non-carbon Miras following the same relation at $K$ (Feast, MN, in press). The more luminous red varlables seem to fall into at least two distinct groups which have been interpreted as core helium (or carbon) burning superglants and AGB stars. The amplitudes appear to be smaller in the first group than the second (ApJ 272, 99; 273, 116). The large amplitude variables of high luminosity seem to show a P-L relation which is steeper than that of the Mira variable (Lloyd Evans, MN, in press). Catchpole and collaborators (SAAO) are continuing infrared observations of the more luminous MC red variables.

\section{$\underline{\text { V. Clusters }}$}

The large young clusters in the $M C$ are of great importance in the study of the evolution of massive stars. NGC 1866 in particular with Its Cepheids is a vital cluster with which to compare evolutionary predictions. Such comparisons (ApJ 270, 155; PASP 95, 122) suggest $(Y, Z$ age $)=\left(0.27,0.016,86 \times 10^{6} \mathrm{yr}\right)$. NGC 1856 is of similar age (ApJ 276,509) and also contains Cepheids, NGC 1847 is $-25 \times 10^{6}$ years old (PASP 95, 5). Ages of $-10^{\circ}$ years have been derived for NGC $2133,2134,2058$ and 2065 (PASP 96, 28; 94, 894) and $[\mathrm{Fe} / \mathrm{H}]--1.0$ was found for the first two clusters. For a number of late-type supergiants in young MC clusters, infrared photometry shows that the LMC ones are metal deficient by -3 compared to the Galaxy with the SMC superglants more deficient. End-main-sequence masses of $-14 M$ are estimated (ApJ 277,149 ). There is a suggestion of radial colour varlation 8 in LMC young populous clusters possibly due to delayed star formation in the cluster cores (AA $110,348)$. Data suggest that the cluster ma in sequences are much wider than predicted by conventional theory (AA 108, 145). Strömgren photometry has been used to derive metallicities of stars in one MC young cluster. The young cluster NGC 330 (SMC) has $[\mathrm{Fe} / \mathrm{H}]=-1.8$. Results in the LMC suggest a range of abundances at a given age (AA 119,75$)$.

The current situation concerning intermediate and old clusters in the MC may be summarized as follows. c-m diagrams of 6 LMC clusters all indicate ages less than $10^{\circ}$ years and a significant population of populous clusters older than this is unlikely (ApJ 278, 582). Analys is of integrated spectra leads to similar conclusions (ApJ 261, 85; see also Astrophys Sp Sc 83, 143). For one cluster in the 
above sample an estimate of $[\mathrm{Fe} / \mathrm{H}]=-1.3$ was made (ApJ 275, 15). Faint photometry indicates that NGC 1466, NGC 2210 and NGC 2257 (LMC) are genuinely old clusters (like galactic globular clusters) (ApJ 266, 82; PASP 95, 967; see also Walker, MNASSA, in press).

The age and metallicity of old and intermediate age MC clusters has been studied on the basis of JHK photometry of cluster members (ApJ Sup 48, 161; ApJ $263,629)$. The age distribution of LMC clusters peaks at $-4 \times 10^{9}$ years. Also, in the LMC, there is an age-metallicity relation though with an (apparentiy significant) scatter. Spectrophotometry of cluster stars yields similar results (ApJ 258, 143). Simple models of chemical evolution are adequate to $f 1 t$ the observed LMC age-abundance correlation (see also ApJ 256, 447). Attempts to bring all the age estimates onto the same system (Hodge, ApJ 264, 470) suggest that ages based on bolometric luminosities of $C$ star members may be in error due to uncertainties in the theory. There may be different radlal distributions of $H B$ stars, glants and subgiants in the old LMC cluster NGC 1806 (Ast Sp Sc 84, 133).

The kinematics of clusters in the LMC younger than $10^{9}$ years are similar to those of young objects (HI gas HII regions, etc.). Older clusters appear to belong to a different system which however is also disc-like. There is no evidence for a kinematic halo amongst LMC clusters (ApJ 272, 488; 259, 89). Velocitles and metallicities for 3 SMC globular clusters have been derived (ApJ Sup 55, 45). Radi1, structural parameters and luminosity functions for SMC clusters are published (AA Sup 49, 1; 52, 143; AA 108, 344). Integrated magnitudes and colours of $55 \mathrm{MC}$ clusters have been determined (PASP 95, 461). Bright clusters in the LMC have greater ellipticities than fainter ones. A correlation between ellipticity and age has been suggested but this needs to be disentangled from the correlation with luminosity (cf. S108, PASP 95, 839; MN 199, 565; AA 125, 359; Obs 102, 228).

\section{Stellar Content}

Cataloguing of the contents of the MC is basic to MC research. The following catalogues have been published: (1) About 200 late $K$ and early $M$ supergiants in the SMC (AA Sup 53, 255); (2) 839 late type supergiants in the LMC (with B, V, R) (AA Sup 51,277 ) (in (1) and (2) the frequencies of variables are estimated); (3) 524 SMC members (with UBV) (AA Sup 50, 291); (4) UBV for 245 LMC stars (AA Sup 50, 7); (5) an addendum to non-supergiants near MC (AA Sup 57, 169); (6) Identification charts for LMC stars (AA Sup 48, 1); (7) a comparison of the catalogue of Stockt (LMC) with other work (Bull Cen Don Stell No. 22); (8) objective prism radial velocities of 418 LMC members and 1127 foreground stars (AA Sup 48, 409) (extensive CORAVEL velocities have also been obtained for MC red supergiants). Photoelectric sequences are: (1) A UBVRI sequence in the SMC bar (AA Sup 50, 119); (2) extens ion of the LMC Bok-Tifft LMC sequence (AA 114, 213); (3) three LMC cluster sequences (MN 197, 693).

Blanco and McCarthy summarized results of their major survey for $C$ and $M$ stars in the MC. The numbers of $C$ stars in the LMC and SMC are in proportion to the masses of the systems (AJ 88,1442 ). The $C / M$ ratio has been discussed as a metallicity index (ApJ 263,259). The existence of M giants on two distinct AGB's in the LMC suggests two discrete epochs of star formation (ApJ 274, L57). Infrared scans show that objects in the LMC with $M_{\text {pol }}\left\langle-6.4, \mathrm{~T}_{\mathrm{BB}}\right\rangle 700 \mathrm{~K}$ must be very rare (ApJ 275,84$)$. Faint photographic photometry suggests B $_{\text {hat }}$ even $9 \mathrm{kpc}$ from the LMC bar the main population is of intermediate age (ApJ Sup 55, 127), and similarly for the western periphery of the SMC (MN 198, 935) and in the LMC bar (ApJ 278, 592, Ardeberg ${ }^{+}$, in progress). Courtest have obtained $M C$ isophotes at $8500 \mathrm{~A}$ from the Salyut-7 satellite. From Spacelab-1 experiments they find a UV stellar cloud which appears to belong to the LMC/SMC bridge (Science 225, 179; COSPAR 1984).

Rocca-Volmerange+ (AA 104, 177) discuss MC star formation rates. They $\mathrm{find}$ a 
similar IMF as in the solar neighbourhood and (contrary to some other work) a smooth rate of evolution. However evidence for stochastic star formation has been given (AA 131, 347; 123, 121; 110,54). Star formation in 30 Dor may have been triggered by the large scale compression of gas moving around the LMC bar. Studies of large gas "bubbles" and associated $\mathrm{OB}$ associations in the LMC lead to an age-diameter correlation (AA 127, 113).

Humphreys has analysed the differences between the distribution of the brightest supergiants in the HR diagrams of the SMC and LMC (ApJ 265, 176). The infrared properties of normal and peculiar A supergiants in the SMC are similar (Obs. 104, 193). Further evidence for metal deficiencies in SMC supergiants has been given (ApJ 265, 716; AA Sup 48, 375).

Magellanic Cloud supergiants are important sources of information on stellar winds and mass loss rates. Mass loss rates in excess of $10^{-4} \mathrm{Mo} / \mathrm{yr}$ were found for LMC F-G supergiants (PASP 93, 567). In MC early type superglants the strength of the stellar wind is generally less than in comparable galactic stars (ApJ 255, 70 ; 256, L49; ApJ Sup 55, 1). Observations over a wide wavelength range have been used to derive effective temperatures and radil for early type MC supergiants ( $M N$ 200, 55; 205, 231; ApJ 255, 70; ApJ Sup 55, 1). The effects of circumstellar absorption or MC 0-type stars is small (AA 91, 53; of. also AA 115, 97; 117, 53) though some examples of circumstellar dust have been found (ApJ Sup 55, 1).

Current results on WR stars (IAU Symposium 99; ApJ Sup 51, 505) do not indicate major global differences between MC WR stars and those in the Galaxy (AA Sup 54, 229; ApJ 268, 228). Despite similar spectral appearances LMC early WN stars span 3 mags in Mv (ApJ 264, 126; cf. also PASP 95, 411). Masses have been derived from several WR binaries (ApJ 257, 110; 257, 116; see also AA 103, 83; 102, 401). Ring nebulae round WR stars may perhaps be regarded as supergiant planetaries (ApJ $256,452 ; 255,79 ; 269,202)$. Sanduleak's star has a nitrogen over-abundance suggesting a similarity to $n$ Car (ApJ 275, 584). S22 (LMC) may have an accretion disk (AA 126, 427). Shell ejection from the carbon star HV2379 has been suggested (MN 202, 31p) though the interpretation is not entirely straightforward (Feast+, MN, in press).

\section{Distance, Structure, Magellanic Stream}

The distances of the MC are important in their own right and also for extragalactlc distance scale determinations ( $\$ 108$, C82). Feast (MN, in press) has summarized recent evidence for the LMC (true) modulus and $f$ inds 18.5. An estimate of $18.3+0.2$ has been obtained from $G$ supergiants (AJ 88, 1187). A value of 19.1 was found from OB stars for the SMC (PASP 94, 31). SMC Cepheids indicate a very large tilt of the SMC to the line of sight (BVI, Caldwell and Coulson; JHK, Stoble and Laney) with a resulting large range of distances. The suggestion (Mathewson and Ford S108) of a division of the SMC into two distinct bodies may be too simple a picture. This structure may be related to the Magellanic Stream (see also M31, MN 205,773 ). Structure of Magellanic type galaxies is summarized by Feitzinger (Ast Sp Aci Rev 27, 35).

The possibility of a gaseous halo in the direction to the MC has been discussed. Some workers believe that the observations indicate a halo to the MC whilst some belleve the results should be interpreted differently. Others have suggested that this line of sight through the halo of our Galaxy is not typical of the entire galactic halo (ApJ 243,460;248, 945;257, 587; 267, 93; 248, 956; MN 204, 317; 205, 1191). Fehrenbach and Terzan (AA Sup 55, 103) find the density of foreground high velocity stars towards the MC twenty times greater than in other directions of similar galactic latitude. Perhaps this is connected with Saha's result from RR Lyraes that the galactic halo does not have spheroidal symmetry (Frascati Workshop on Pop II Variables, 1984). 


\title{
8. WORKING GROUP ON INTERNAL MOTIONS IN GALAXIES (S.T. Gottesman)
}

\begin{abstract}
Studies of internal motions in galaxies are tabulated below; $R, O$, and $T$ refer to radio, optical, and theoretical investigations. Three general references are of interest: IAU Symposium No. 100 (Reidel, 1983); Mor phology and Dynamics of Galaxies (Geneva Observatory, 1982); Instrumentation in Astronomy $\underline{V}$ (SPIE, 1983).
\end{abstract}

Object First Author Reference Object First Author Reference

\begin{tabular}{|c|c|c|c|c|c|}
\hline NGC 157 & Zasov & 029.158 .044 & NGC 185 & Johnson & R 34.157 .188 \\
\hline NGC 205 & Johnson & R 34.157 .188 & NGC 221 & Pellet & 031.158 .087 \\
\hline NGC 221 & Bendinelli & T 33.157 .160 & NGC 224 & Sawa & $\operatorname{RT} 31.158 .101$ \\
\hline NGC 224 & Linke & R 31.158 .348 & NGC 224 & Sofue & o 32.158 .158 \\
\hline NGC 224 & Sawa & R 32.158 .051 & NGC 224 & Bajaja & R 32.158 .005 \\
\hline NGC 224 & Lawrie & 034.157 .111 & NGC 224 & McElr oy & 034.157 .028 \\
\hline NGC 224 & Unwin & R 34.157 .078 & NGC 224 & Unwin & R 34.157 .079 \\
\hline NGC 224 & Boulanger & R 29.158 .004 & NGC 225 & Haud & Т 29.158 .078 \\
\hline NGC 253 & Pence & 030.158 .008 & NGC 315 & Dressel & R 33.158 .050 \\
\hline NGC 596 & Williams & o 29.158 .084 & NGC 598 & Boulesteix & 0 In Prep. \\
\hline NGC 613 & Blackman & O 29.158 .130 & NGC 613 & Peterson & 0 In Prep. \\
\hline NGC 628 & Shostak & R AA 132,20 & NGC $62 \overline{8}$ & Briggs & R 32.158 .092 \\
\hline \multirow[t]{2}{*}{ NGC 672} & Carozzi - & & & & \\
\hline & Meyssonler & 031.158 .050 & NGC 891 & vanderkru1t & T 29.158 .285 \\
\hline NGC 891 & Solomon & R 31.158 .342 & NGC 925 & Marcel in & 031.158 .110 \\
\hline NGC 936 & Kormendy & O 34.157 .187 & NGC 1055 & Barabanov & O 29.158 .139 \\
\hline NGC 1058 & vander Kruit & R AA 134,258 & NGC 1068 & Scoville & R 34.158 .049 \\
\hline NGC 1068 & Galletta & OT 32.158 .044 & NGC 1079 & Gallagher & OR33.157.035 \\
\hline NGC 1097 & Wolstencroft & O 29.158 .112 & NGC 1097 & Me aburn & 029.158 .033 \\
\hline NGC 1097 & Blackman & 029.158 .130 & NGC 1275 & van Corkom & R 33.158 .108 \\
\hline NGC 1313 & Marcelin & o 31.158 .014 & NGC 1313 & Blackman & o 29.158 .130 \\
\hline NGC 1316 & Bosma & 0 In Press & NGC 1365 & Phillips & 033.158 .066 \\
\hline NGC 1365 & Jorsater & 0 Preprint & NGC 1365 & Phillips & o 32.158 .287 \\
\hline NGC 1365 & Blackman & 029.158 .130 & NGC 1553 & Kormendy & 0 In Press \\
\hline NGC 1566 & Comte & O AA 114,7 & NGC 1569 & Young & R ApJ276,476 \\
\hline NGC 1961 & Shostak & R 32.158 .248 & NGC 1961 & Gottesman & T MN $202,21 p$ \\
\hline NGC 2525 & Blackman & 031.158 .011 & NGC 2655 & Hucht meier & R 31.158 .305 \\
\hline NGC 2685 & Richstone & 032.158 .040 & NGC 2712 & Krumm & R 32.158 .286 \\
\hline NGC 2715 & Huchtme ier & R 31.158 .305 & NGC 2814 & Andreasyan & o 33.157 .131 \\
\hline NGC 2841 & Young & R 32.158 .105 & NGC 2903 & Marcelin & 034.157 .084 \\
\hline NGC 3031 & Bash & T 30.158 .010 & NGC 3031 & Pellet & 031. \\
\hline NGC $3031 \mathrm{~d}$ & Sargent & R 33.157 .027 & NGC 3034 & Stark & R 31.158 .347 \\
\hline NGC 3034 & Olofsson & R AA 136,17 & NGC 3067 & Rub in & OR31.158.141 \\
\hline NGC 3115 & Ill Ingworth & 031.158 .156 & NGC 3200 & Rub in & o 33.157 .127 \\
\hline NGC 3310 & Galletta & OT32.158.044 & NGC 3359 & Gottesman & R 31.158 .327 \\
\hline NGC 3504 & Peterson & 032.158 .007 & NGC 3628 & Tacconi & R 31.158 .344 \\
\hline NGC 3690 & Fischer & I 34.157 .106 & NGC 3726 & Mey sonnier & 0 In Press \\
\hline NGC 3729 & Schwarz & $\mathrm{R}$ In Press & NGC 3783 & Pelat & O 29.158 .147 \\
\hline NGC 3810 & Mey sonnier & 0 In Press & NGC 3893 & Mey sonnier & 033.157 .113 \\
\hline NGC 3938 & vander Kruit & R 31.158 .079 & NGC 3992 & cottesman & R 32.158 .094 \\
\hline NGC 3992 & Gottesman & $\mathrm{R}$ In Press & NGC 3998 & Blackman & 033.158 .035 \\
\hline NGC 3998 & Knapp & $\mathrm{R}$ In Press & NGC 4151 & Schulz & 0 In Press \\
\hline NGC 4203 & Burstein & R030.158.206 & NGC 4278 & Gallagher & O 29.158 .245 \\
\hline NGC 4359 & Meysonn ier & 0 In Press & NGC 4594 & Bajaja & 0 In Press \\
\hline NGC $4650 \mathrm{~A}$ & Schechter & O АрJ 277,526 & NGC 4730 & Pellet & 031.158 .087 \\
\hline NGC 4731 & Gottesman & R Prepr int & NGC 5055 & Johansson & $\mathrm{R}$ In Prep. \\
\hline NGC 5128 & M\&̈1lenhoff & 029.158 .00 & NGC 5128 & Graham & 030.158 .033 \\
\hline NGC 5128 & Craham & 033.158 .175 & NGC 5128 & Marcel in & 033.158 .062 \\
\hline
\end{tabular}


NGC 5128 NGC 5194 NGC 5194 NGC 5194 NGC 5236 NGC 5363 NGC 5457 NGC 5457 NGC 5813 NGC 5905 NGC 5908 NGC 6081 NGC 6221 NGC 6503 NGC 6946 NGC 6946 NGC 7013 NGC 7331 IC 342 IC 342 IC 1727

IC 5063

UGC 7576 VV 48 VV 574 VII 2W403 3C 218

\section{Mark 10}

\section{Mark 266}

Mark 538

Vela Ring

Fornax Cohen

Pks0349-27Danz Iger Monnet

Carozz1- van der Hulst $R 33.158 .011$ Hjalmarson $R$ In Prep.

O 30.158 .137

Scoville R 33.157.013

deVaucouleurs 034.157 .048

Haynes R 29.158 .248

Solomon $\quad R \quad 31.158 .345$

Bosma R 29.158.006

Kormendy 0 In Press

van Moorsel R 31.158.092

van Moorsel R 31.158.092

Williams R 34.157 .126

Pence $\quad 033.157 .257$

deVaucouleurs 032.158 .114

Young $\quad \mathrm{R} \mathrm{32.158.011}$

Winnberg $R$ In Prep.

Knapp R AA 133,127

Young R 32.158.105

Young R 32.158.011

Lo $\quad R$ ApJ 282,L59

Meyssonier 031.158 .050

Danziger $\quad 030.158 .014$

Schechter R MN 208,111

Arkhipova $\quad 033.157 .005$

Arkhipova $\quad 034.157 .021$

OR29.158.231

R033.158.012

O AA 115,209

O 29.158 .027

o 32.158 .085

O MN 208,601

o 34.157 .030

o MN 208,589
NGC 5173

NGC 5194

NGC 5194

NGC 5236

NGC 5301

NGC 5383

NGC 5457

NGC 5728

NGC 5813

NGC 5907

NGC 5963

NGC 6118

NGC 6240

NGC 6503

NGC 6946

NGC 6946

NGC 7252

NGC 7479

IC 342

IC 694

IC 2082

UGC 7576

UGC 12423

VV 219

II ZW 73

Hydra

3C 293

Mark 79

Mark 348

Cartwheel Davies

AM064-741 Few
$\mathrm{R}$ In Press

$\mathrm{R}$ In Press

R 31.158 .343

O 29.158 .236

R 32.158 .286

OT 33.157 .128

R 33.157 .051

R 34.157 .054

o 32.158 .267

RT33.157.118

O 31.158 .295

0 In Press

O AA 118,166

O 33.157 .215

R 31.158 .340

O 32.158 .168

0 31.158 .007

o In Prep.

R 31.158 .340

I 34.157 .106

0 29.158 .037

o 32.158 .104

R 32.158 .017

o 33.157 .005

R MN 208,111

R033.158.012

R 29.158 .074

O AA 115,209

R 31.158 .143

o 31.158 .128

0 32.158 .271

031.158 .145

Seyfert Galaxies Tohline T 31.158 .009 Mirabel R ApJ 277,92 Shuder $\quad 032.158 .028$

Active and Radio Galaxies $\begin{array}{ll}\text { Bieging } & \text { R } 33.158 .033 \\ \text { Shostak } & 033.158 .054 \\ \text { Heckman } & \text { R } 33.158 .115\end{array}$

Elliptical Calaxies

Sharples $\quad 033.157 .020$

Copacciol1 T MN 209,317

Illingworth $\quad 033.157 .029$

Dressel1 R 32.158.029

Tonry $\quad 030.158 .213$

Tonry $\quad 029.158 .243$
Afanas'ev
Bieging
Dibaj

Jenk ins

Jenk ins

Brosche

Brosche

Davies

Knapp

Bertola

Dressler

Giovanardi
○ 30.158 .038

R 30.158 .004

o 31.158 .045

O MN 207, 361

0 30.158 .018

T 33.157 .109

T 33.158 .117

033.157 .046

R 32.158 .336

o 30.158 .101

Spheroidal/SO Galaxies

$\begin{array}{ll}\text { Hawarden } & \text { OR29.158.282 } \\ \text { Schechter } & \text { O } 34.157 .041 \\ \text { Lentes } & \text { T P Stat Meth ESA 1983, } 73\end{array}$

Early Type Galaxies Dav1s

029.158 .010

Disk Galaxies Kormendy

o 33.157 .024

Binney
Mirabel

Kormendy

Kormendy
O 33.157 .026

R 34.157 .124

R 34.157 .029

O ApJ 256,460

0 ApJ 265,632 


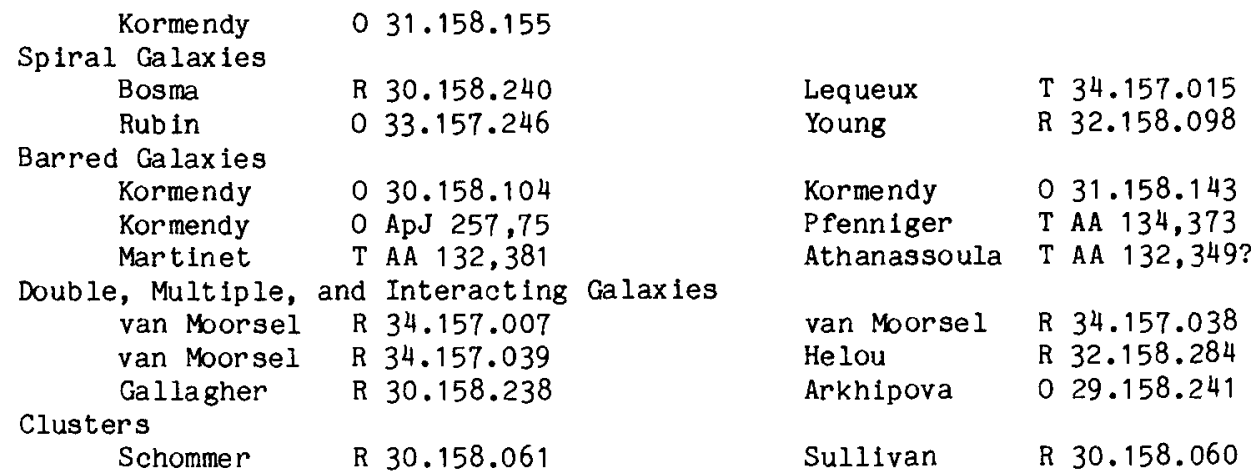

Nuclear Emission cD Galaxies

Irregular Galaxies Superthin Galaxies Face On Galaxies Galaxy Kinematics Galaxy Dynamics HI in Galaxies Sa Calaxies Sab Galaxies Sb Galaxies Sc Galaxies

$\begin{array}{lll}\text { Vernon } & 0 & 30.158 .021 \\ \text { Valentijn } & \mathrm{R} & 32.158 .170 \\ \text { Hunter } & 0 & 32.158 .097 \\ \text { Goad } & \text { OR } & 30.158 .173 \\ \text { Rickard } & \mathrm{R} & 30.158 .167 \\ \text { Athanassoula } & \text { ORT } & 30.012 .004 \\ \text { Illingworth } & \mathrm{O} & 30.158 .102 \\ \text { Sancisi } & \mathrm{R} & 30.158 .106 \\ \text { Rubin } & 0 & \text { In Press } \\ \text { Elmegreen } & \mathrm{R} & 31.158 .322 \\ \text { Rubin } & 0 & 32.158 .215 \\ \text { Burstein } & \mathrm{T} & 31.158 .024\end{array}$

9. THE WORKING GROUP ON GALAXY PHOTOMETRY AND SPECTROPHOTOMETRY (J.-L. Nieto)

This triennium (the period mid-1981 to Oct. 1984) ended with a Colloquium "New Aspects of Galaxy Photometry" held in Toulouse on Sept. 1984, cover ing recent technological improvements and their application to star formation, galactic dynamics and galactic evolution. Related to galaxy photometry and spectrophotometry is IAU Symposium 110 "Internal kinematics and dynamics of galaxies" (33.012.004) and IAU Colloquium 78 "Astronomy with Schmidt-type telescopes" (Aug. 1983).

Three major books have been published: "The Revised Shapley-Ames Catalogue of Galaxies by Sandage and Tammann (30.002.091); "The ESO/Uppsala Survey of the ESO (B) Atlas" (Lauberts, 31.002.098); "The Atlas of Selected Galaxles" by Takase, Kodaira and Okamura (University of Tokyo Press), and a useful tool: "Detailed Bibliography on Surface Photometry" by Davoust and Pence (32.002.003) which is being updated.

\section{Optical, UV, and IR Surface Photometry}

LUMINOSITY STANDARD NGC 3379. Ratcliff and Burstein (32.158.337), Nieto (34.157.006 and 34.157.014), Capaccioli and de Vaucouleurs $(34.157 .042)$ for profiles, Nieto and Vidal for geometry (AA 135, 190) and center (MN 209, 21P), Bendinel11+ (AA 140, 174) for nucleus.

NEARBY GALAXIES. M31 (Hodge and Kennicutt, 31.158.072; Hiromoto+, 34.157.087; Ann and $\mathrm{Yu}(31.158 .158$ with also M106 and M33); Innanent, 31.158.044), M51 (Monnet+, 30.158.137; Smith, 32.158.217), Leo II DG (Hodge, 32.158.338), NGC 205 (Price and Grasdalen, 34.157.189), DDO 155 (de Vaucouleurs and Moss, 34.157.033). 
EARLY-TYPE GALAXIES. M87, NGC 2300, NGC 2768 with a use of a CCD in the scanning mode by Boroson + (34.157.123), NGC 3379 (see above), NGC 404 and NGC 524 (Barbont, 32.158.250), NGC 1600 and 4261 (Barbont, AA 137, 166), S0s with polar rings (A0136-081, Schweizert, 34.157.001; NGC 3384, Davoust+, MN 209, 503), several Es and SOs (Souviron, thesis, University of Par1s 7), NGC 5253 (Morwood and Glass, 32.158.245), NGC 3557 (fast rotating elliptical, Capacciolit, AA 135, 89), ellipticals with shells (a catalogue by Malin and Carter, 34.157.147; NGC 1344, Cartert, 31.158.058; NGC 5128, Malin +, 34.158.077); dust lane ellipticals (survey by Longmore and Sharples, 32.158.149; ellipticals with ionized gas disks and search for [OII] by Caldwell (ApJ 278, 96 and PASP 96, 287); NGC 4696, Jorgensent, 33.157.219; IC 4320, Warren-Smith and Berry, 34.157.080; IC 5063, Appenzeller and Coida, 30.158.139; NGC 5128, Harding ${ }^{+}, 30.158 .219$, and Adams + , 33.158.019; NGC 6251, Nietot, 33.158.067; Cygnus A, Thompson (ApJ 249, L47)), dark halo around NGC 1199 (Walker and Andersen, preprint), halo of M87 (Boughn and Saulson, 33.157.028), and correlation between photometric parameters (Thomsen and Frandsen, 33.157.228).

SPIRAL GALAXIES. 25 objects by Boroson (30.158.059), 34 non-barred and 15 barred by Elmegreen and Elmegreen (ApJ Suppl 54, 127 and in preparation, with a near infrared atlas, 30.002.073), several barred spirals by Baumgart (thesis, $U$. of Missour 1), 16 galaxies by Wevers, Ph.D. thesis (University of Coningen), NGC 5963 and 16 low surface brightness spirals (Romanishint, 31.158 .295 and 34.157.049), NGC 253 (Pence, 30.158.008), NGC 1313 (Marcellin and Athanassoula, 31.158.014), Sculptor Group Galaxies (Carlgnan, preprint), NGC 5383 (Duval and Athanassoula, 33.157.128), 109 field galaxies (Kent, preprint), NGC 7479 (Blackman, 33.157.021 and Benedict, 31.158.056), NGC 1566 (Hackwell and Schwelzer, 33.157.025), NGC 4501 (Send, 32.158.042), NGC 7331 (Telescot, 32.158.258), NGC 7814 and 5746 (Jonest, 34.157.024), NGC 4156 (Nieto and Tiennot, AA 131, 291), NGC 1079 (Gallagher and Bushouse, 33.157.035), face-on spirals by van der Kruit and Shostak (NGC 3938, 31.158 .079 ; NGC 628, AA 132, 20; NGC 1058, AA 134, 258), inner region of NGC 3351 (Allo in and Nleto, 32.158.274), of NGC 2997 (Meaburn and Terrett, 31.158.315), halo of NGC 253 (Beck+, 31.158.084), a comparative study of spiral bulges with pure spheroidal galaxles (W1rth and Shaw, 30.158.063, 33.157.037); galactic disks as exponentials (Seiden+, ApJ 282, 95).

EDGE-ON GALAXIES. NGC 5907, 5023, 4217, 4013 (van der Kruit and Searle, $31.158 .308)$, NGC 7814 (31.158.309), NGC 4762 and other galaxles by Hamabe, Okamura and colleagues $(31.158 .099,31.158 .284,32.158 .211)$, NGC 4762 by Hamabe and Wakamatsu (in press), UGC 7576 (Mould+, 32.158.104), NGC 4565 (Boughn +, 30.158.176, Jensen and Thuan, 32.158.307), NGC 7172 (Sharplest, MN 208, 15), discussion on the width of the dust by Hacke+ $(32.158 .167)$.

PECULIAR GALAXIES. NGC 7252 (Schwelzer, 31.158.007), NGC 6240 (Fried and Schulz, 33.158.016), NGC 4656-4657 (Staytont, 33.157.168), UGC 6697 (Gavazz1+, AA 137, 235), NGC 1316 and 1052 (Cartert, 34.158.055), NGC 3448 (Bertolat, AJ 89, 350), Mrk 325 (Coup inot+, 31.158.144; Dettmart, AA 130, 424), nebulosities surrounding active nuclei (Weistropt, 30.158 .152 and 34.158 .063 ; Hutchingst, $32.141 .059,32.141 .131$ ApJ 280, 41); Malkant (ApJ 280,66); Wyckof $f+, 30.141 .023$, 33.159.008; McKenty and Stockton (ApJ 283, 64); Mrk 421 and 521, Hicksont, 31158.293; 20 Mrk Seyferts, (Yee, 34.158.087), interacting objects (NGC 5426-5427, Blackman, 32.158.035; IC 5174/75, West and Barbier, 31.158.083; IIZw40, Baldwint, 31.158.012; AM 064-741, Few+, 31.158.145; VV371, Scholl and Grayzeck, PASP 96, 216; Arp 91, Jenkins, ApJ 277, 501 and others, Joseph+, MN 209, 111), Haro galaxies (KInman and Hintzen, 31.158.123), others (Brosch, 32.158.046; Barbler1+, 32.158.081; Wakamatsu, 33.157.237; Wakamatsu and Arp, 33.157.103; Sulentic, 33.158.026; Talent+, 32.158.102; Bertola and Sharp, MN 207, 47; Romanishin, PASP 96, 24; Kinney+, PASP 96, 398; Morwood and Glass, AA 135, 281), Mrk or UV-excess objects (often with spectrophotometry) (Mi tchell, 32.158.347; Takase +, 34.157.141; Petrosyant, 34.158.093, etc. See reports on active galaxies and on research in USSR). 
DWARF ELLIPTICAL OR COMPACT GALAXIES. Virgo cluster (Caldwell, 34.157.229; Bothun and Caldwell, ApJ 280, 528; Vigrouxt, preprint; a catalogue by Reaves, 34.002 .052 ), in the M81/M82 group (Börngen and Karachentseva, 32.158 .165 and 32.158.244), near NGC 1023 (Davies and Kinman, MN 207, 173), with near IR photometry (Gondhalekar, MN 209, 59), a JHK survey (Thuan, 33.157.122), central region of NGC 1510 (Eichendorf and Nieto, AA 132, 342), others (Börngen and Kalloglyan, 32.158.013).

CD GALAXIES. 7 X-ray galaxies by Valentijn (33.157.019), giant ellipticals in clusters (Thuan and Romanishin, 30.158.049, Morbey and Morris, 34.160.102), VZw311 (Schneider and Gunn, 32.160.058), cluster brightest members (Murphy +, 33.160.019; Schneider+, 33.160.007 and 33.160.082).

GALAXIES IN CLUSTERS. 20 galaxies in Virgo and 261 in Virgo and Ursa major (Watanabe+, 32.160.026; 33.157.255; 34.157.150), 84 Virgo galaxies (Fritze, $32.158 .166), 81$ Virgo disk systems (Borosont, 34.160.097), 109 galaxies in Virgo cluster center to serve as standards (Bingelilt, AJ 89,64), as well as a classification scheme and an Atlas of dwarf galaxies (Sandage and Bingelli, AJ 89, 919). IR survey of 53 virgo spirals (Scovillet, 34.157.046), edge-on spirals in Ursa Major and Cancer (Weekes, 30.158.169), E's and SO's in Fornax, Virgo and Sersic 129-1 (Griersmith, 31.160.033), in Abell 370 and Cl 2244-02 (Bautz+, $31.160 .016)$, in $0016+16$ (Koo; 30.160.048), 17 intermediate redshift clusters (Butchert, 34.160.006), Abell 1367 (Godwin and Peach, 32.158.037), Abell 1775 (Richter and Schnell, 30.158.156).

LINE PHOTOMETRY. Spirals in three clusters by Kennicutt+ (AJ 89, 1279), narrow-band photometry of dominant cluster galaxy by Heckman+ $(30.158 .002)$, cores of $X$-ray clusters by Cowlet $(34.160 .065)$.

NUCLEI AND/OR CENTRAL REGIONS. Center of M31 (Kent, 33.157.049), of M32 (Tonry, ApJ 283, L27), nucleus of M33 (Nieto and Aur iere, 31.158.319; Gallagher+, 32.158.257), central region of NGC 3379 (see above), elliptical galaxies (Lauer, preprint), nuclear structure of M87 (Zavatti+, AA 134, 99), cDs (Kron and Albert, 32.158 .350 ), (near) IR photometry of the nuclei of NGC 5128 (Lepine+, AA 131, 72), NGC 253 (Beck and Beckwith, MN 207, 671), of normal spirals (Willnert, PASP 96, 143), barred spirals (see above).

ULTRAVIOLET PHOTOMETRY. 13 ellipticals with ANS (Astronomical Netherlands Sateliite) (de Boer, 32.158.228), of M101 (Steckert, 31.158 .076 and Hil1+ ApJ 277, 542 ), of the nucleus of M83 (Bohlin, 34.157.151), of Virgo galaxies (Smith and Cornett, 32.160.025) and energy distribution of spiral and irregular galaxies (Code and Welch, 31.158.094) and of M31 and M33 (Davist, 31.158.327).

IRAS results on M31 (Habing+, ApJ 278, L59), Shapley-Ames galaxies (de Jong+, ApJ 278, L67), infrared galaxies (Solfer+, ApJ 278, L71), the Hercules cluster (Young+, ApJ 278, L75), Arp 220 (Soifert, ApJ 283, L1). IR survey by Lee $(31.158 .059)$, by Heckmant $(34.158 .086)$, two-band photometry of 3664 faint galaxies by Hutchins $(33.157 .174)$.

OPTICAL POLARISATION. M 82 (AxOn, 30.158.046).

\section{Geometry, Morphology and Technical Aspects}

Elliptical galaxies (Leach, 30.158.051), variation of geometry (Gamaleldin and Issa, 33.157.052), orientation of the plane of M33 (Maucherat+, AA 133, 341), isophotometry of the Leo group (Dickson and Hodge, 30.160.009), inclination and brightness (Arreguine and Sersic, 34.157.145), diameters of rings of spirals (Pedreros and Madore, 30.158.024), standard $D_{25}$ for coma galaxies (Paturel, 31.158 .052 ), for 237 galaxies (Fouque and Paturel, 34.157.013), 500 southern 
late-type galaxies (Corwint, 31.158.055), objective and/or quantitative classifications systems (Whitmore, ApJ 278, 61; Okamura+, ApJ 280, 7 ).

Discussions on Zwicky's magnitudes (Bothun and Schlommer, 31.158.067; Aumant, 31.158.077; Giovanelli and Haynes, AJ 89, 1; de Vaucouleurs and Corwin, in preparation), on faint galaxy photometry from ground-based telescopes and ST (Tyson, PASP 96, 566), estimates of reddening for galaxies in catalogs (Burstein and Heiles, ApJ Suppts 54, 33), influence of seeing (Ellist, 34.036.038; Peterson and Collins, 34.158.003; Disney and Phillips, 34.157.113; Djorgovsk1, J Astr Astr 4,271 ), calibration of photographic plates (Feitzingert, 34.036.037), automatic classiflcation of spiral galaxies (Schlosser and Musculus, 33.036.088; Thonnat, 34.036.204), shape analysis (Pasian and Santin, preprint), digital treatment (Sulent1c and Lorre, 33.160.027), deconvolution of photographic images from PSF (Bendinellit, AA 138, 337), detection of faint objects with photographic and computer techniques (Couch+, MN 209, 307), systematic and accidental errors (Capaccioli and de Vaucouleurs, 34.157.042), influence of ellipticity on profiles (Nieto, 33.158.053), accuracy from quick derivation of profiles (Whitmore and Kirschner, 31.158.142); see also workshops on Astronomical Measuring Machines (Sept 1982, 33.012.059) and on Astronomical Photography (April 1984).

\section{Multiaperture Photoelectric Photometry}

A major event is the publication of the general catalogue of photoelectric magnitudes and colors in the UBV system of 3578 galaxies brighter than the 16 th $\mathrm{V}$ magnitude (1936-1982) by Longo and de Vaucouleurs $(34.002 .060)$. A similar catalogue for infrared bands is in preparation ( 1600 galaxies). Also appeared the third catalogue of spectroscopic and photometric observations of galaxles from the ESO/Uppsala list (West+, 30.002.046), a similar catalogue for galaxies from the Horologlum region (Chincarinit, AA Suppls Ser 57, 1) and an extensive survey of 248 $E$ and So southern galaxles (Sadler, AJ 89, 34).

UBV PHOTOMETRY. 36 Mrks and SOs (Petersont, 30.158.055), 61 bright galaxies (Stone, 31.158.289), disk galaxles near clusters (Bothunt, 31.158 .323 and 31.158.324), 46 Es and SOs (Michard, 32.158.003), early-type galaxies with known line widths (de Vaucouleurs, Corwin and Frueh, in preparation), galaxies in the direction of the Hercules supercluster (Buta and Corwin, in preparation), six possible Seyfert (Kinman, 33.158.017), 163 DDO dwarf galaxies (de Vaucouleurs+, 30.158.170 and 33.157.227), 8 Es and SOs by Dottorit (33.157.154), 105 double galaxies $(30.002 .049), 74$ southern galaxies $(32.1580 .006)$, brightest ellipticals by Jackson (Ph.D. thesis, U. Callf. Santa Cruz); 6724 galaxies in the fleld of Coma (Godwint, 33.160.013).

BVR of early-type cluster galaxies (Lugger, ApJ 278, 51) UBVRI of 28 Mrk galaxies (Huttert, 30.158.225), 300 nearby galaxies (Corwin and Smyth, in preparation), VRI of Virgo and field spirals (Visvanathan, 30.158.030), $g$ and $r$ of 48 galaxies in clusters (Melnick and Quintana, AJ 89, 1288), UBVJKH of early type spirals (Grlersmith+, 32.158.018), intermediate band filter spectrophotometry with 10 filters for 56 galaxies (Soleim+, 31.158 .300 and 32.158.272), IR multiaperture photometry for isolated galaxies (Brosch and Isaacman, 32.158.082).

\section{Spectrophotometry}

This technique has been applied extensively to active galaxies, whose report is presented elsewhere. We shall concentrate here on normal or mildly active galaxies (galaxies studied also photometrically are included in section A).

NUCLEI. M51 (Rose and Searle, 31.158.033; Rose and Cecil, 33.157.047), nucleus of M33 (O'Connell, 33.157.120), Mi01 (as well as 5 HII regtons) (Rayot, 31.158.065), NGC $7793\left(D_{1 a z}+32.158 .224\right.$ and 33.158.023), 93 remote galaxies (Keel, 
$34.157 .005), 116$ nuclei (Feldmant, 31.158.153).

HII REGION NUCLEI. NGC 1097 (Talent, 31.158.179), NGC 1097 and NGC 1598 (Phililips+, MN 210,701), NGC 1365 (Alloint, 30.158.135; Edmunds and Pagel, 31.158 .019 ) and five others (Phillipst, MN 207, 25).

ELLIPTICAL GALAXIES. Population models (Gunn+, 30.158.154), NGC 4388 (Phillips and Malin, 31.158.311), NGC 3344 (McCall+, 30.158.054), IC 5063 (Danziger+, 30.158.014), NGC 6946 (de Glola-Eastwood+, ApJ 278, 564), 12 metal poor dwarf galaxies (Kunth and Sargent, 34.157.252), IZw36 (V1allegond and Thuan, 33.157.240), clumpy irregulars (Boesgaard+, 31.158 .008 and Duflot-Augarde and Allo in, 32.158.043), irregular galaxies (Huntert, 31.158 .290 and ApJ 276, L35), NGC 3660 (Kollatschny+, 33.157.056), NGC 3690 (Gehrz+, 33.157.162), NGC 5128 (Graham and Price, 30.158.033), NGC 1275 (Wirtht, 33.158.170), NGC 4449 (Sabbadint, AA 131, 1), interacting galaxles (Kennicutt and Keel, ApJ 279, L5; Bushouse and Gallagher, PASP $96,273)$.

SPECTROPHOTOMETRY OF HII REGIONS IN GALAXIES. We restrict the 1 ist to the studies of Zinn (Ursa Major DG, 30.158.212), of Bouleste1x+ (M33, 30.158.216), of Kennicutt and Kent (200 field and Virgo cluster galaxies, 34.157.003). Mapping of IIne emission in some galaxies can be found in Fabiant (32.158.152), Comte and Duquennoy (32.158.169), Marcellin and Condoin (33.157.055), Graham (30.158.196), Courtest $(30.158 .031)$, Will laamst (ApJ 281, 571).

IUE OBSERVATIONS. UV data on the following (non active) objects: M31 (Deharvengt, 31.158.082; Welch, 32.158.030), M33 (Clanit, AA 137, 223), NGC 4649 (Bertolat, 31.158.041), M81 (Bruzual+, 32.158.103), NGC 4594, 3031, 5194, 4258 (Ellist, 32.158.150), clumpy irregulars (Benvenutit, 31.158.017), NGC 4214 and 4670 (Huchra+, 34.157.146), isolated galaxies (Broscht, AA 135, 330).

\section{THE WORKING GROUP FOR SPACE SCHMIDT SURVEYS}

(K. Henize)

This Working Group was formed at the 1976 Grenoble IAU General Assembly to coordinate the interests and efforts of those astronomers who feel the growing need for a large-aperture (-one meter), wide-field, fast-focal-ratio space telescope. The basic objectives of such a telescope are: 1) to conduct a Palomar-quality sky survey in the far UV, 2) to detect and study very faint extended sources which, because of field diameter and focal ratio constraints, are very difficult to study with presently planned space telescopes, and 3) to permit objective prism/grating spectroscopy in the far UV. In particular, it is expected that this telescope would serve the Space Telescope in the same way the Palomar Schmidt served the 200-inch telescope - by detecting those stars and galaxies with special or anomalous characteristics in UV wavelengths which would be of interest to observers using the Space Telescope.

Studies directed at the design and use of a Space Schmidt telescope during the past three years have been carried out primarily at the Asiago Observatory/Padova University in Italy, at the Observatoire de Mareseille/CNRS Laboratoire d'Astronomie Spatiale in France, and at the Naval Research Laboratory (NRL) in the USA.

IAU Colloquium 78, "Astronomy with Schmidt-type Telescopes" (L. Rosino, chairman) was held at Aslago Observatory in August 1983. This colloquium reviewed the many uses of Schmidt telescopes and included a session pointing out the needs for a large-aperture Schmidt telescope in space. There were 120 participants from 27 countries in attendance. In early 1984, F. Bertola, in collaboration with M. Golay and $R$. West, submitted to ESA a statement of the Space Schmidt concept in 
response to a call for mission concepts for future ESA planning.

Lemaitre at the Observatoire de Marsellle continues optical studies of the all-reflecting Schmidt telescope and maintains a laboratory capable of producing large aspher ic mirrors of the type required as the corrector for all-reflecting Schmidts. He presented a paper entitled "Optical Design with the Schmidt Concept: 1. Ground-based Development, 2. The Space Schmidt Project for the 1990's?" at IAU Colloquium 78 .

Carruthers and Page have completed their analysis of data obtained with the S201 electrographic Schmidt camera operated on the moon during the Apollo 16 mission. A summary, "The S201 Far Ultraviolet Imaging Survey: A Summary of Results and Implications for Future Surveys" (PASP 96, 447, 1984), contains much information related to the conduct of UV surveys. The NRL group is preparing to utilize the electrographic Schmidt camera on two future space missions. The Mark I camera (used for the S201 program), modifled to utilize a microchannel intensifier stage, will be used in the Space Test Program to study diffuse nebulae and to measure the galactic UV background. It is estimated that this camera will be 100 times more sensitive to diffuse sources than the original S201 camera. A related instrument, the Mark II version of the electrographic Schmidt camera, will be flown on the Spartan 3 Shuttle sub-satellite. This instrument has an aperture of $15 \mathrm{~cm}, \mathrm{a}$ resolution of 30 arcsec over an eleven-degree field and is expected to reach a limiting UV magnitude $\left(M_{1400}\right)$ of 15.

Carruthers and Heckathorne have continued studies of large-format, highresolution electrographic cameras suitable for use as the detector in a Space Schmidt telescope. Henize, using data from the Skylab S019 UV spectrograph, has derived a spectrum classification system for $O$ and early-B stars using the very strong C IV and Si IV lines in the far ultraviolet (AJ 86, 1658, 1981). This system gives sensitive discrimination of luminosity among the 0-stars and of temperature for the late-0 and early-B stars. Since it utilizes the strongest absorption lines in the UV/optical region of the spectrum, such a system should be of interest in studying the $O$ and early-B stars in nearby galaxies via objective-prism spectroscopy with a Space Schmidt telescope.

\section{SUPERNOVA WORKING GROUP (V. Trimble)}

The Supernova Working Group currently consists of a mailing list of about 150 professional and amateur astronomers from 18 countries, who engage in supernova searches, observations, and interpretation, elther through their own research or through their institutional positions or IAU commission responsibilities. Not all are IAU members. An official chairman and membership list will be established at the XIVth General Assembly. The Working Croup communicates via a semi-annual newsletter. Contributions from anyone who has items of interest to those engaged in supernova research would be welcome.

SEARCHES. IAU Circulars carried 83 reports of new SNe between 1981 January 1 and 1984 September 1 , including a handful of false alarms and simultaneous independent discoveries. Of the reports, 36 came from Maza and his colleagues at Cerro el Roble, Chile; 12 from Terebizht at the Sternberg Crimean Station; 8 from Rev. Robert Evans at Maclean, NSW; 6 each from Wild + at Berne and Lovas ${ }^{+}$at Konkoly Observatory, Budapest; 3 from ESO; 2 each from As iago and Okazaki at Yamagata, Japan; and 1 each from the Harvard Agassiz Station, Palomar (Arp), Westerbork, Moore Observatory, Abastumani (Kimeridze), Itajuba Brasil (Pellegrini), CIT (Kowal), and Las Campanas (Tammann). 
Of the automated searches that have been on the horizon for several years, only that of Cawson (Cambridge UK; U. Arizona) using rejected UK Schmidt plates and the Cambridge Automatic Plate Measuring system has found any supernovae. Three other projects at U. Arizona (McGraw), UC Berkeley (Muller and Pennypacker), and New Mexico (Colgate and Pearce) have hardware and software substantially complete and have begun recording reference images. All expect to begin searching regularly during winter 1984-85.

OBSERVATIONS. Recent new kinds of data include (a) the discovery of radio emission from the Type I event 1983n in M83 (Sramek, Panagla and Weiler, 1984 ApJ Lett, in press; radio emission had previously been seen only from Type II events), which has been interpreted by Chevalier (1984 ApJ Lett, in press) as requiring a binary progenitor of initial masses $8+6.5 \mathrm{M}$ and (b) the first pre-maximum spectrum of a Type II event, 1983k in NGC 4699 (Nlemela, Rulz, and Phillips 1984, preprint), which shows high-ionization NIII and HeII emission lines, atop a strong blue continuum, somewhat reminiscent of Wolf-Rayet stars, a possible class of SNII progenitor. Finally, Cadonau and Tammann (Basel) are engaged in a study of SNI light curves that will probably rule out a division into fast and slow subclasses.

PROGENITORS AND MODELS. Most workers continue to bet on core collapse in massive single stars as the underlying energy source for Type II's and some form of nuclear detonation or deflagration following mass transfer onto a white dwarf in a close binary system for Type I's. The most serious problem for the SN II's has been transferring enough energy to the stellar envelope to eject it at the observed speeds. In the 8-10 M range, recent models by Nomoto (Tokyo) and Hillebrandt (Munich) Indlcate that a shock wave set of $f$ by core bounce can be fed enough energy by burning of oxygen in intermediate, infalling layers to keep going and eject material. In the 10-30 $\mathrm{M}$ range, Wilson (Lawrence Livermore) finds that significant neutrino heating occurs at late times (after a second rather than a millisecond) and so re-energizes stalled shocks, yielding the requisite ejection.

For the SN I's, problems remain in matching the products of the nuclear explosions to the compositions implied by observed spectra and in identifying sufficient numbers of progenitor systems. For instance, Branch (Oklahoma) finds that a standard deflagration model (Nomoto, Thlelemann, and Wheeler, 1984, ApJ 279 , L23) reproduces observed abundances only if products from all the layers of the exploding star are well mixed before we see them, so as to yleld roughiy solar composition (apart from the total absence of $\mathrm{H}$ ). The problem of identifying enough SNI progenitors (Observatory 102, 133, 1982) becomes rather worse if white dwarfs in typical novae eject more than they accrete and so erode away into AM CVn-like objects (Nather, Texas). Both difficulties are considerably alleviated if the immediate progenitor is a close pair of degenerate dwarfs that spiral together in something like the Hubble time (Paczynski in "Seventh North American Workshop on Cataclysmic Variables and Low-Mass X-Ray Binaries", January 1983). Detai led discussions of the scenario have been given by Webbink (1984 ApJ 277, 355) and Iber and Tutukov (1984 ApJ Sup 54, 335).

DISTANCE INDICATORS. The 45 year history of the use of supernovae as standard candles (Wilson $1939 \mathrm{ApJ} 90,634$ ) has been a patchy one. Recently, Her shkowitz, Linder, and Wagoner (Stanford, preprint) have concluded that the proper allowance for scattering in the low-density atmospheres of Type II events reduces the emergent flux (for a given, measured color temperature) sufficiently below black body levels to imply a Hubble constant, $\mathrm{H} \geq 104 \mathrm{~km} \mathrm{~s}^{-1} \mathrm{Mpc}^{-1}$. For Type I events, on the other hand (Suther land and Wheeler 1984, ApJ 280, 282; Arnett, work in progress), simultaneous matching of light curves and spectra by nuclepar explosion models can only be achleved for $\mathrm{H}_{0}$ in the range 36 to $72 \mathrm{~km} \mathrm{~s}^{-1} \mathrm{Mpc}^{-1}$. 